\title{
X-Ray Polarimetry with the Polarization Spectroscopic Telescope Array (PolSTAR)
}

Henric S. Krawczynski ${ }^{\mathrm{a}}$, Daniel Stern ${ }^{\mathrm{b}}$, Fiona A. Harrison ${ }^{\mathrm{c}}$, Fabian F. Kislat ${ }^{\mathrm{a}}$, Anna Zajczyk ${ }^{\mathrm{a}}$, Matthias Beilicke $^{\mathrm{a}}$, Janie Hoormann ${ }^{\mathrm{a}}$, Qingzhen Guo ${ }^{\mathrm{a}}$, Ryan Endsley ${ }^{\mathrm{a}}$, Adam R. Ingram ${ }^{\mathrm{d}}$, Hiromasa Miyasakac $^{c}$, Kristin K. Madsen ${ }^{\mathrm{c}}$, Kim M. Aaron ${ }^{\mathrm{b}}$, Rashied Amini ${ }^{\mathrm{a}, \mathrm{b}}$, Matthew G. Baringe, Banafsheh Beheshtipour $^{\mathrm{a}}$, Arash Bodaghee ${ }^{\mathrm{f}}$, Jeffrey Booth ${ }^{\mathrm{b}}$, Chester Borden ${ }^{\mathrm{b}}$, Markus Böttcher ${ }^{\mathrm{g}}$, Finn E. Christensen $^{h}$, Paolo S. Coppi ${ }^{i}$, Ramanath Cowsik ${ }^{\mathrm{a}}$, Shane Davis ${ }^{\mathrm{j}}$, Jason Dexter ${ }^{\mathrm{k}}$, Chris Done ${ }^{\mathrm{l}}$, Luis A. Dominguez ${ }^{\mathrm{b}}$, Don Ellison ${ }^{\mathrm{m}}$, Robin J. English ${ }^{\mathrm{b}}$, Andrew C. Fabian ${ }^{\mathrm{n}}$, Abe Falcone ${ }^{\mathrm{o}}$, Jeffrey A. Favretto $^{\mathrm{b}}$, Rodrigo Fernández ${ }^{\mathrm{p}, \mathrm{q}}$, Paolo Giommi ${ }^{\mathrm{r}}$, Brian W. Grefenstette ${ }^{\mathrm{c}}$, Erin Kara ${ }^{\mathrm{n}}$, Chung H. Lee $^{\mathrm{b}}$, Maxim Lyutikov ${ }^{\mathrm{s}}$, Thomas Maccarone ${ }^{\mathrm{t}}$, Hironori Matsumoto ${ }^{\mathrm{u}}$, Jonathan McKinney ${ }^{\mathrm{v}}$, Tatehiro

Mihara $^{w}$, Jon M. Miller ${ }^{\mathrm{x}}$, Ramesh Narayan ${ }^{\mathrm{y}}$, Lorenzo Natalucci ${ }^{\mathrm{z}}$, Feryal Özel ${ }^{\text {aa }}$, Michael J. Pivovaroff $^{\text {ab }}$, Steven Pravdo ${ }^{b}$, Dimitrios Psaltis ${ }^{\text {aa }}$, Takashi Okajima ${ }^{\text {ac }}$, Kenji Toma ${ }^{\text {ad }}$, William W. Zhangac

${ }^{a}$ Washington University in Saint Louis, Physics Department and McDonnell Center for the Space Sciences, Saint Louis, MO 63130, USA

${ }^{b}$ Jet Propulsion Laboratory, California Institute of Technology, Pasadena, CA 91109, USA

${ }^{c}$ Cahill Center for Astronomy and Astrophysics, California Institute of Technology, Pasadena, CA 91125, USA

${ }^{d}$ Anton Pannekoek Institute for Astronomy, Science Park 904, P.O. Box 94249, 1090 GE Amsterdam, The Netherlands

${ }^{e}$ Rice University, Department of Physics and Astronomy, P.O. Box 1892, Houston, TX 77251-1892, USA

${ }^{f}$ Department of Chemistry, Physics, and Astronomy, Georgia College, Milledgeville, GA 31061, USA

${ }^{g}$ Centre for Space Research, North-West University, Private Bag X6001, Potchefstroom, 2520, South Africa

${ }^{h}$ DTU Space, National Space Institute, Technical University of Denmark, Elektrovej 327, DK-2800 Lyngby, Denmark

${ }^{i}$ Department of Astronomy, Yale University, P.O. Box 208101, New Haven, CT 06520-8101, USA

${ }^{j}$ Department of Astronomy, University of Virginia, P.O. Box 400325, Charlottesville, VA 22904-4325, USA

${ }^{k}$ MPI for Extraterrestrial Physics, Giessenbachstr. 85748, Garching, Germany

${ }^{l}$ Centre for Extragalactic Astronomy, Department of Physics, Durham University, South Road, Durham, DH1 $3 L E$

${ }^{m}$ Department of Physics, North Carolina State University, 400L Riddick Hall, Raleigh, NC 27695-8202, USA

${ }^{n}$ Institute of Astronomy, Madingley Road, Cambridge CB3 OHA, UK

${ }^{\circ}$ Penn State University, Department of Astronomy and Astrophysics, 516 Davey Lab, University Park, PA 16802, USA

${ }^{p}$ Department of Physics, University of California, Berkeley 94720, CA, USA

${ }^{q}$ Department of Astronomy \& Theoretical Astrophysics Center, University of California, Berkeley CA 94720, CA, USA

${ }^{r}$ ASI Science Data Center, Via del Politecnico s.n.c. I-00133 Rome Italy

${ }^{s}$ Department of Physics and Astronomy, 525 Northwestern Avenue, West Lafayette, Indiana 47907-2036, USA

${ }^{t}$ Texas Tech University, Physics Department, Box 41051, Lubbock, TX 79409-1051, USA

${ }^{u}$ Nagoya University, Center for Experimental Studies, Kobayashi-Maskawa Institute for the Origin of Particles and the Universe, Furo-cho, Chikusa-ku, Nagoya 464-8602, Japan

${ }^{v}$ University of Maryland, Physics Department, College Park, MD 20742-4111, USA

${ }^{w}$ RIKEN, 2-1 Hirosawa, Wako, Saitama, Japan 351-0198

${ }^{x}$ Univ. of Michigan in Ann Arbor, Astronomy Dept., 830 Dennison, 500 Church St., Ann Arbor, MI 48109-1042, USA

${ }^{y}$ Harvard-Smithsonian Center for Astrophysics, 60 Garden Street, MS-51, Cambridge, MA 02138, USA

${ }^{z}$ Instituto di Astrofisica e Planetologia Spaziali, INAF, Via Fosso del Cavaliere 100, Roma I-00133, Italy

${ }^{a a}$ Department of Astronomy/Steward Observatory, 933 North Cherry Avenue, Tucson, AZ 85721-0065, USA

${ }^{a b}$ Lawrence Livermore National Laboratory, Livermore, CA 94550, USA

${ }^{a c}$ NASA Goddard Space Flight Center, Greenbelt, MD 20771, USA

${ }^{a d}$ Astronomical Institute, Tohoku University, 6-3 Aramaki, Aoba-Ku, Sendai, Japan, 980-8578 
Abstract: This paper describes the Polarization Spectroscopic Telescope Array (PolSTAR), a mission proposed to NASA's 2014 Small Explorer (SMEX) announcement of opportunity. PolSTAR measures the linear polarization of $3-50 \mathrm{keV}$ (requirement; goal: 2.5-70 keV) X-rays probing the behavior of matter, radiation and the very fabric of spacetime under the extreme conditions close to the event horizons of black holes, as well as in and around magnetars and neutron stars. The PolSTAR design is based on the technology developed for the Nuclear Spectroscopic Telescope Array $(N u S T A R)$ mission launched in June 2012. In particular, it uses the same X-ray optics, extendable telescope boom, optical bench, and CdZnTe detectors as NuSTAR. The mission has the sensitivity to measure $\sim 1 \%$ linear polarization fractions for X-ray sources with fluxes down to $\sim 5$ mCrab. This paper describes the PolSTAR design as well as the science drivers and the potential science return.

Keywords: X-Ray Polarimetry; Astronomical Instrumentation; Black Holes; Neutron Stars; Blazars; General Relativity.

\section{Introduction}

In the following, we describe the Polarization Spectroscopic Telescope Array (PolSTAR), a satellite-borne experiment measuring the linear polarization of X-rays in the energy range from 3-50 keV (requirement; goal: 2.5-70 keV). The mission was proposed to NASA's 2014 Small Explorer (SMEX) announcement of opportunity (National Aeronautics and Space Administration, 2014). The mission concept builds on the highly successful Nuclear Spectroscopic Telescope Array $(N u S T A R)$ hard X-ray imaging mission (Harrison et al., 2013). The main difference between Pol$S T A R$ and NuSTAR is the addition of a scattering element in the focal plane of the X-ray telescope enabling the measurement of the linear polarization properties. PolSTAR measures the polarization fraction and angle, two properties of photon beams characterizing the uniformity and orientation of the electric field carried by the photons, respectively, as a function of photon energy and arrival time. The two fundamentally new observables depend on the emission mechanism, scattering angles, and the geometry and properties of matter, electromagnetic fields and spacetime itself of extreme objects such as black holes and neutron stars. A mission like PolSTAR gives geometric information about objects which are much too small to be imaged directly. For example, consider the Galactic stellar mass black hole GRS 1915+105. At a distance of $\sim 8.6 \mathrm{kpc}$ (Reid et al., 2014), the gravitational radius $r_{\mathrm{g}}=G M / c^{2}$ (with gravitational constant $G$, black hole mass $M$, and speed of light $c$ ) measures $21 \mathrm{~km}$, corresponding to an angle of 4.5 femto-degrees. X-ray polarimetry allows us to measure angles in systems of such small angular extent.

$\mathrm{X}$-ray polarimetry is a largely unexplored field. NASA has so far only launched one dedicated Xray polarimetry mission, $O S O-8$, which was in orbit from 1975 to 1978 (Novick, 1975). OSO8 measured the polarization fraction and angle of the $2.6 \mathrm{keV}$ and $5.2 \mathrm{keV}$ X-ray emission from the Crab Nebula (Weisskopf et al., 1978) and set upper limits on the polarization fraction of the Xray emission from 14 sources (Silver et al., 1979; Hughes et al., 1984).

Two instruments on the International GammaRay Astrophysics Laboratory (INTEGRAL), the Spectrometer on INTEGRAL (SPI; Vedrenne et al., 2003) and the Imager on Board the $I N$ TEGRAL Satellite (IBIS; Ubertini et al., 2003), have revealed tentative evidence for highly polarized $>100 \mathrm{keV}$ emission from the Crab Nebula (Dean et al., 2008; Forot et al., 2008) and Cyg X-1 (Laurent et al., 2011; Rodriguez et al., 2015), albeit with large systematic errors. Several authors reported the detection of highly polarized $\mathrm{X}$-ray and/or gamma-ray emission from gammaray bursts (GRBs; e.g., Willis et al., 2005; McGlynn et al., 2007; Kalemci et al., 2007; Yonetoku et al., 2011, 2012; Kostelecký and Mewes, 2013, and references therein), but the evidence is not highly significant taking the statistical and systematic errors into account.

PolSTAR uses scattering off a lithium hydride $(\mathrm{LiH})$ element to measure the linear polarization of X-rays. In the $2-10 \mathrm{keV}$ energy band, a compet- 


\begin{tabular}{|l|l|l|}
\hline Parameter & Requirement & Current Best Estimate \\
\hline Telescope bandpass (keV) & $3-50$ & $2.5-70$ \\
\hline $\begin{array}{l}\text { Telescope effective area (effective \# of } \mathrm{NuS} \text { - } \\
\text { TAR optics) }\end{array}$ & $\geq 0.9$ & 1.1 \\
\hline Energy resolution (FWHM at 6 keV) & $\leq 1 \mathrm{keV}$ & $0.45 \mathrm{keV}$ \\
\hline Absolute timing accuracy (msec) & $\leq 15$ & 2 \\
\hline $\begin{array}{l}\text { Angular resolution (half power diameter; arc- } \\
\text { sec) }\end{array}$ & $\leq 80$ & 60 \\
\hline $\begin{array}{l}\text { Pointing, during science portion of orbits } \\
(99.7 \% \text { CL) }\end{array}$ & $\begin{array}{l}\leq 62^{\prime \prime} \text { from stick } \\
\text { center }\end{array}$ & $17^{\prime \prime}$ from stick center \\
\hline $\begin{array}{l}\text { Instrument reconstructed pointing knowl- } \\
\text { edge (99.7\% CL) }\end{array}$ & $\leq 15^{\prime \prime}$ & $8^{\prime \prime}$ \\
\hline $\begin{array}{l}\text { Minimum Detectable Polarization (3-15 keV, } \\
25 \text { ks obs'n of 1 Crab source, 99\% CL) }\end{array}$ & $\leq 1 \%$ & $0.5 \%$ \\
\hline $\begin{array}{l}\text { Polarization fraction systematic error } \\
(3-15 \text { keV; 99.7\% CL) }\end{array}$ & $\leq 1.5 \%$ & $0.25 \%$ \\
\hline $\begin{array}{l}\text { Polarization angle systematic error } \\
(\geq 6 \% \text { polarized source; 99.7\% CL) }\end{array}$ & $\leq 20^{\circ}$ & $2^{\circ}$ \\
\hline Bad pixel fraction & $\leq 2 \%$ & $1 \%$ \\
\hline Instrument mass (kg) & $\leq 170$ & 131 \\
\hline Instrument power (W; orbital avg.) & $\leq 45$ & 28 \\
\hline
\end{tabular}

Table 1: PolSTAR has significant margin on all primary instrument requirements, largely based on NuSTAR heritage. CL stands for confidence level.

ing approach uses photoelectric effect interactions in a gas chamber read out by gas electron multipliers (GEMs). The proposed Imaging X-ray Polarimetry Explorer (IXPE; Ramsey, 2014) and $X$ ray Imaging Polarimetry Explorer (XIPE; Soffitta et al., 2013) missions use gas pixel detectors for the readout, enabling spectropolarimetric imaging with an angular resolution of $\sim 25^{\prime \prime}$. The proposed Polarization from Relativistic Astrophysical $X$-raY Sources (PRAXYS) (former GEMS) mission uses a gas chamber operated as a time projection chamber (TPC; Jahoda et al., 2015; Enoto et al., 2014; Hill et al., 2014; Takeuchi et al., 2014; Kitaguchi et al., 2014; Jahoda et al., 2014). The electron track perpendicular to the beam direction are measured in two dimensions based on strip and drift time measurements. PolSTAR is unique in offering a broad energy range, as that of IXPE, XIPE and PRAXYS is limited to 2-10 $\mathrm{keV}$. PolSTAR does not offer the imaging capabilities of IXPE and XIPE. At the time of writing this paper, NASA selected IXPE and PRAXYS for Phase A studies (The National Aeronautics and Space Administration, 2015), and the European Space Agency (ESA) selected XIPE as one of three M4 candidate missions (The European Space Agency, 2015). The NASA review classified PolSTAR as a Category II proposal defined as a "well-conceived and scientifically or technically sound investigations which are recommended for acceptance, but at a lower priority than Category I" attesting to the soundness of the approach. We are currently considering proposing an enlarged version of PolSTAR to NASA's upcoming Medium Class Explorer (MIDEX) announcement of opportunity.

The paper is structured as follows. After presenting the PolSTAR design in $\S 2$ and the analysis methods and projected performance in $\S 3$, we discuss the PolSTAR science program in $\S 4$. Section $\S 5$ gives a summary.

Unless otherwise noted, all figures and sensitivity estimates assume source fluxes normalized to the observed time-averaged $2-12 \mathrm{keV}$ fluxes measured from 1996-2011 with the All-Sky Monitor (ASM; Levine et al., 1996) on the Rossi X- 


\begin{tabular}{|l|l|l|}
\hline Parameter & NuSTAR & PolSTAR \\
\hline $\begin{array}{l}\text { \# telescope mod- } \\
\text { ules }\end{array}$ & 2 & 1 \\
\hline $\begin{array}{l}\text { Effective focal } \\
\text { length }\end{array}$ & $10.14 \mathrm{~m}$ \\
\hline Optics & $\begin{array}{l}\text { Grazing incidence, } \\
\text { (conical approx.) }\end{array}$ \\
\hline $\begin{array}{l}\text { \# shells per op- } \\
\text { tics module }\end{array}$ & 133 \\
\hline $\begin{array}{l}\text { Multilayer coat- } \\
\text { ing }\end{array}$ & $\mathrm{W} / \mathrm{Si}$ and Pt/C & $\mathrm{W} / \mathrm{Si}$ \\
\hline Detectors & $32 \times 32$ pix CZT hybrids \\
\hline$\#$ detectors & 8 & 17 \\
\hline Shielding & CsI anti-coincidence \\
\hline
\end{tabular}

Table 2: Based largely on NuSTAR heritage, the PolSTAR instrument is $82 \%$ build-to-print by mass.

ray Timing Explorer ( $R X T E$; Jahoda et al., 1996) mission (from R. Remillard, private communication). All statistical errors are given as $1 \sigma$ errors (68.7\% confidence level, CL). As systematic errors tend to exhibit non-Gaussian distributions, we provide them as $99.7 \%$ CL (equivalent to $3 \sigma$ for a Gaussian distribution). We follow the standard in the field to give fluxes in units of mCrab (milli-Crab), equaling $1 / 1000^{\text {th }}$ of the flux from the Crab Pulsar and Nebula. See Meszaros et al. (1988), Lei et al. (1997), Costa et al. (2006), Weisskopf et al. (2006), Bellazzini et al. (2010), and Krawczynski et al. (2011) for reviews of the science drivers and detection techniques of X-ray polarimetry.

\section{Design of PolSTAR}

PolSTAR measures the flux, polarization fraction, and polarization angle of astrophysical sources as a function of energy. Table 1 summarizes the primary PolSTAR instrument requirements and current best estimate (CBE) of capabilities.

As mentioned above, PolSTAR has high heritage. Largely based on $N u S T A R$ (see Harrison et al., 2013, for a description of the flight hardware and its pre-flight and in-flight performance), Pol$S T A R$ uses an identical extendable mast, structures, metrology system, and cadmium zinc tel-

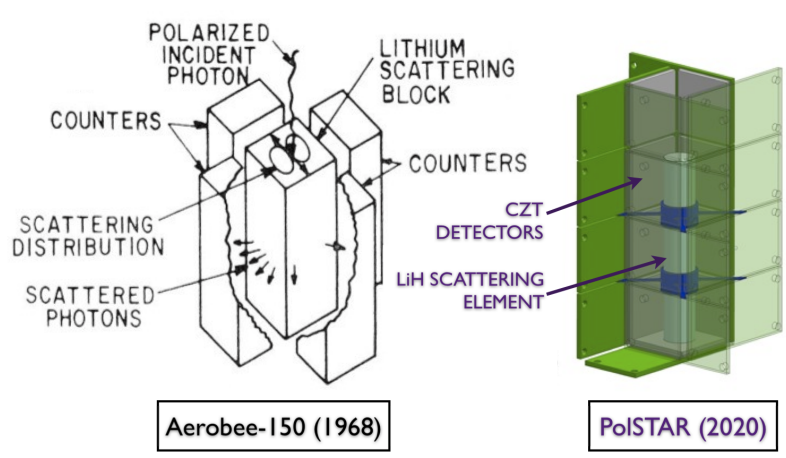

Figure 1: PolSTAR uses the same simple instrument design as the first astronomical X-ray polarimeter, which flew in July 1968 on an Aerobee-150 rocket (Angel, 1969). Fifty years later, PolSTAR can use a similar LiH scattering element design with modern CZT detectors.

luride (CZT) detectors (Table 2). The optics use the NuSTAR design and assembly with simplified $N u S T A R$-heritage coatings. There are three main differences from NuSTAR: first, PolSTAR flies only one telescope rather than two. Second, PolSTAR slowly rotates every 10 minutes to minimize systematic errors on the polarization measurements. Third, PolSTAR inserts a passive scattering element into the light path and arrays the CZT detectors around this element, parallel to the incident photon path, to enable the measurement of the photon polarization; NuSTAR uses the same CZT detectors perpendicular to the incident photon path to provide focused images of the high-energy sky. The PolSTAR detection principle is very simple, essentially identical to that of the very first astronomical X-ray polarimeter, an experiment flown on an Aerobee-150 rocket in July 1968 (Figure 1). Fifty years later, PolSTAR can use modern X-ray optics and more capable detectors to provide the first sensitive polarization measurements of a representative sample of high-energy sources across a broad, scientifically compelling energy range.

\subsection{Detection Principle}

PolSTAR has a 3-50 keV energy range require- 


\begin{tabular}{|l|l|l|}
\hline \multicolumn{2}{|l|}{ Component } & Heritage \\
\hline Optics & Segmented design & NuSTAR \\
\hline & Glass substrates & NuSTAR \\
\hline & Multilayers & NuSTAR \\
\hline & Mounting/assembly & NuSTAR \\
\hline Extendable mast & NuSTAR \\
\hline Metrology systems & NuSTAR \\
\hline Focal Plane & CZT material & NuSTAR \\
\hline & ASIC & NuSTAR \\
\hline & Hybrid sensor & NuSTAR \\
\hline & CsI shield & NuSTAR \\
\hline Polarimeter & LiH scatterer & Aerobee-150 \\
\hline & Design & X-Calibur \\
\hline
\end{tabular}

Table 3: Strong heritage pervades the PolSTAR design.

ment driven by the science objectives, and a 2.5$70 \mathrm{keV}$ capability inherited from NuSTAR. NuS$T A R$ showed that many interesting and diagnostic phenomena uniquely present themselves in this energy range, such as the Compton reflection hump (e.g., Risaliti et al., 2013), and cyclotron absorption lines in neutron stars (e.g., Fürst et al., 2013, 2014a,b, 2015; Tendulkar et al., 2014).

PolSTAR uses X-ray scattering off a passive element to measure the polarization of astrophysical targets. Coherent scattering is the dominant process below $6 \mathrm{keV}$, while at higher energies Thomson scattering and Compton scattering are dominant. Thus, the effective energy range of a scattering polarimeter can be broad, reaching from below a keV to greater than a $\mathrm{MeV}$.

Figures 2 and 3 illustrate how the instrument works. The mirror assembly focuses X-rays onto the passive scattering element, a $6 \mathrm{~cm}$ long, $1 \mathrm{~cm}$ diameter lithium hydride $(\mathrm{LiH})$ cylinder. As photons scatter preferentially perpendicular to the electric field vector, the azimuthal scattering angle distribution can be used to measure the polarization degree and angle (Figure 3). The scattered photons are photo-absorbed in the assembly of CZT detectors that surround the LiH. These detectors, identical to those on $N u S T A R$, register each incident photon with a time, energy, and location. The intensity as a function of azimuthal scattering angle constrains the polarization fraction and angle, and this is done as a function of energy.
It is instructive to compare the design of Pol$S T A R$ to that of other scattering polarimeters, i.e. NASA's balloon-borne X-Calibur hard Xray polarimeter experiment (Krawczynski et al., 2011; Guo et al., 2013; Beilicke et al., 2014a,a) and the soft gamma-ray telescope (SGT) of JAXA's ASTRO-H mission (Fukazawa et al., 2014). Whereas PolSTAR uses a passive lowatomic number (low- $Z$ ) scattering element, $X$ Calibur and the SGT use active scattering elements made of heavier elements. PolSTAR's passive $\mathrm{LiH}$ scattering element enables polarimetric measurements in the key 2.5-30 keV energy range. The active scattering elements of $X$-Calibur and the SGT result in a much higher energy thresholds ( $\sim 30 \mathrm{keV}$ for $X$-Calibur and $\sim 50 \mathrm{keV}$ for the SGT), but enable additional background suppression capabilities through the coincident detection of the Compton scattered photon and the Compton electron. In the case of the SGT, the active scattering elements enables furthermore an improved energy resolution by measuring the energy given to the Compton electron. One reason for the higher energy threshold of active scattering elements is the higher $Z$ of active detector elements. $X$-Calibur uses the scintillator EJ-200 which contains roughly equal amounts of $\mathrm{H}(Z=1)$ and $\mathrm{C}(Z=6)$, and the SGT uses Si $(Z=14)$ pad detectors. The heavier elements exhibit a much lower scattering efficiency than $\mathrm{LiH}$ owing to the prevalence of photoelectric effect absorption over scattering interactions. More quantitatively, the energy at which the scattering cross section starts to dominate above the photoelectric absorption cross section is $9 \mathrm{keV}, 20 \mathrm{keV}$, and $80 \mathrm{keV}$ for $\mathrm{LiH}$, $\mathrm{C}$ and $\mathrm{Si}$, respectively. The requirement to trigger the active scattering elements elevate the energy threshold for polarimetric studies even more. Using the standard Compton equations, and assuming a trigger threshold of $2 \mathrm{keV}$ for the $X$-Calibur scintillator and $5.4 \mathrm{keV}$ for the SGT Si pad detectors, we infer effective energy thresholds of 33 $\mathrm{keV}$ and $55 \mathrm{keV}$ for $X$-Calibur and the SGT, respectively. Note that $X$-Calibur is optimized for operation on a balloon. As the residual atmosphere at a flight altitude of 125,000 feet anyhow absorbs $<30 \mathrm{keV}$ photons, an active scintillator 


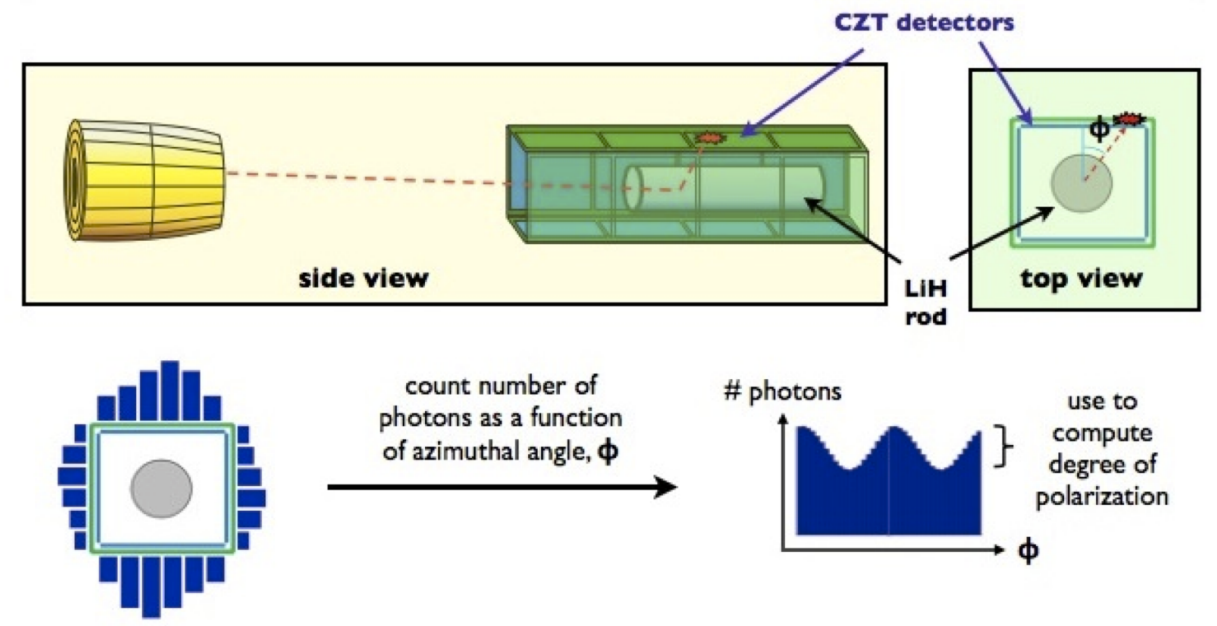

Figure 2: How PolSTAR works: photons are focused by the grazing incidence optics onto the LiH scattering rod, where they preferentially scatter perpendicular to the electric field vector. Four columns of four CZT detectors surround the rod to detect scattered photons. A $17^{\text {th }}$ detector at the far end of the scattering element provides a source image. PolSTAR measures the polarization fraction and angle by measuring the azimuthal distribution of scattered photons.

scattering element is a good choice for $X$-Calibur.

PolSTAR achieves excellent energy resolutions although it does not measure the energy of the Compton electrons. The main reason is that 2.5$70 \mathrm{keV}$ photons loose only a small fraction of their energy when Compton scattering. For example, a $E_{\gamma}=10 \mathrm{keV}$ photon scattering by $\theta=90^{\circ}$ gives only $E_{\mathrm{e}}=\left(1-\left(1+E_{\gamma} / m_{e} c^{2}\right)^{-1}\right) E_{\gamma} \approx 0.2 \mathrm{keV}$ to the Compton electron (see $\S 3.3$ and Fig. 10).

\subsection{Instrument Subsystems}

Table 3 summarizes the heritage of the Pol$S T A R$ instrument components. PolSTAR can reuse a large fraction of the NuSTAR hardware and software. The most significant changes with respect to NUSTAR are the introduction of the passive $\mathrm{LiH}$ scattering element and the slow rotation of the satellite. The scattering element has heritage from the Aerobee-150 rocket experiment (Angel, 1969) and the more recent X-Calibur balloon experiment. Below we discuss key instrument subsystems in more detail.

Grazing incidence optics: The PolSTAR optics are a simplified version of the NuSTAR grazing incidence optics, fabricated by the same per- sonnel using the same equipment. The reflecting surface of each glass substrate is coated with a depth-graded multilayer consisting of up to several hundred alternating thin layers of high and low index of refraction material. The small reflections from each layer add in phase, achieving a broad bandpass over a relatively large field-ofview. PolSTAR uses NUSTAR bilayer thickness recipes, deposited using the same custom, highthroughput planar magnetron sputtering facility at DTU-Space (Denmark's national space institute) as used by NuSTAR. As shown by Sanchez Almeida and Martinez Pillet (1993), grazing incidence optics produce negligible instrumental polarization $(<0.1 \%)$. The PolSTAR grazing incidence optics contain 133 nested multilayer-coated shells in a conical approximation to a Wolter-I geometry (Wolter, 1952).

The only change relative to NUSTAR is that $N U S T A R$ used two mirror coating recipes: the inner 89 shells are coated with depth-graded Pt/C multilayers which provide sensitivity up to $79 \mathrm{keV}$ and the outer 44 shells are coated with depthgraded W/Si multilayers which provide sensitivity to $70 \mathrm{keV}$. PolSTAR only requires an energy 


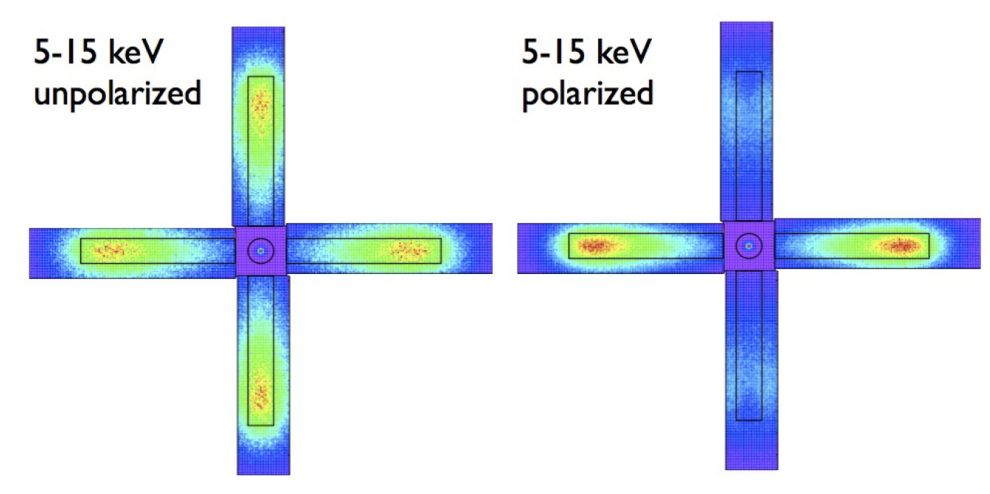

Figure 3: End-to-end Monte Carlo simulations of an unpolarized (left) and polarized (right) source in the 5-15 keV energy range, showing the four unfolded CZT detector modules surrounding the LiH stick, with Detector 17 in the center (see $\S 3$ for details about the simulations). The inset black rectangles and circles indicate the LiH stick size and location. Color scale encodes the number of hits, with red indicating the highest flux. Polarized photons preferentially scatter perpendicular to the electric field vector, creating an azimuthally asymmetric photon distribution.

range of $3-50 \mathrm{keV}$, and therefore uses the less expensive and easier to apply $\mathrm{W} / \mathrm{Si}$ multilayers on all shells. Eliminating the $\mathrm{Pt} / \mathrm{C}$ multilayers also provides a $20 \%$ larger effective area below $50 \mathrm{keV}$ relative to the NuSTAR optics.

The final step in fabricating the nested optic is to mount the glass segments. Alternating layers of precision-milled graphite spacers and glass segments are epoxied together using one of the lathe-like assembly machines procured to build the NUSTAR optics (Figure 4). The final optics module produces an azimuthally symmetric point-spread function (PSF) with a tight $18^{\prime \prime}$ fullwidth at half maximum (FWHM) core and a $58^{\prime \prime}$ half-power diameter (HPD) (Harrison et al., 2013). The NuSTAR PSF varies by $<5 \%$ as a function of energy (Madsen et al., 2015).

Hailey et al. (2010a) give a detailed description of the NuSTAR optics, Zhang (2009) describes the substrate production, Christensen et al. (2011) summarizes the coatings, and the overall optics fabrication is detailed in Craig et al. (2011).

Extendable mast and structures: Pol$S T A R$ uses a build-to-print copy of the canister, deployment mechanism and mast used by $N u S$ $T A R$ to provide the required $10.14-\mathrm{m}$ focal length (Figure 5). These would be fabricated at ATKGoleta using the same team, facility and processes as used for NuSTAR. The flight-validated design provides the required on-orbit stiffness with a near-zero coefficient of thermal expansion (CTE).

PolSTAR uses identical benches and structures to NUSTAR, as well as a mast adjustment mechanism (MAM) at the optics end of the extendable mast that allows tip-tilt adjustment to the optics unit. The MAM provides for on-orbit refinement to the optical axis (Harrison et al., 2013). The NuSTAR benches were designed for two telescopes (i.e., two optics modules, focusing light on two focal plane modules). In order to maximize heritage with NuSTAR to allow for a build-toprint design, and to reduce costs, PolSTAR uses the same benches, but leaves one of the optical arms empty.

Polarimeter: The polarimeter (Figure 6) consists of a LiH scattering element, CZT detectors, readout electronics, and an active CsI shield. The cylindrical $\mathrm{LiH}$ scattering element has a diameter and length of $1 \mathrm{~cm}$ and $6 \mathrm{~cm}$, respectively. $\mathrm{LiH}$ combines a low mean atomic number (implying a high probability for scatterings relative to photoelectric effect interactions) with a relatively high density of $0.82 \mathrm{~g} \mathrm{~cm}^{-2}$ (implying a high interaction probability for a $6 \mathrm{~cm}$ long cylinder). $\mathrm{LiH}$ has flight heritage from being used as a shield on the Department of Energy's Systems Nuclear Auxiliary Power mission. The diameter is driven by the competing desires to have a thin stick to minimize internal absorption, but to have it large compared to the PSF and pointing errors. The current design provides a balance matched to the size of the CZT detector assembly.

$\mathrm{LiH}$ reacts with water and oxygen. The stick would therefore be packaged in a thin Be shield, 


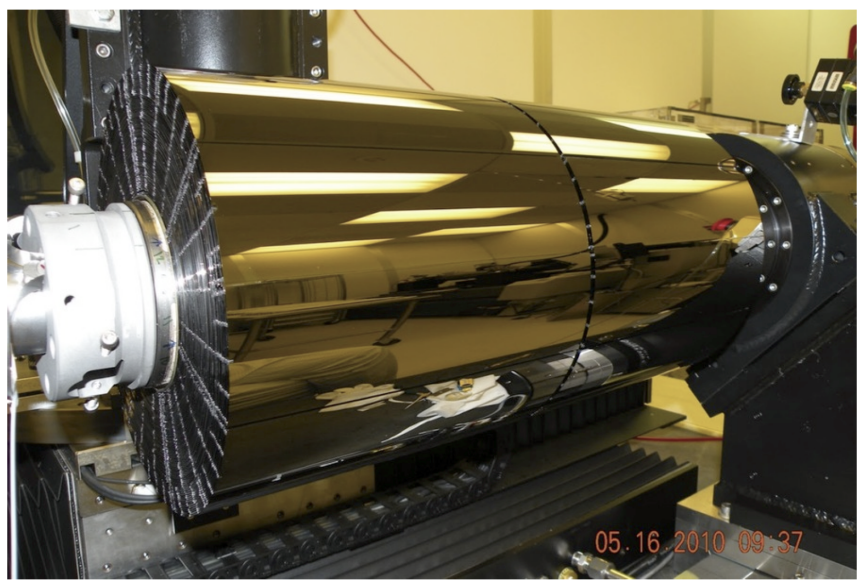

Figure 4: Assembly of the first $N u S T A R$ optics module (FM0), which is essentially identical to the PolSTAR optics module. The optics modules are built up from 133 layers of grazing incidence optics, built up using epoxy and graphite spacers on a computed, numerically controlled (CNC) lathe assembly machine. The optics module is $47.2 \mathrm{~cm}$ long, $19.1 \mathrm{~cm}$ in diameter and weighs $31 \mathrm{~kg}$. This picture, taken on May 16, 2010, shows 82 layers.

$0.5 \mathrm{~mm}$ in thickness along the sides and the rear end, with a $10 \mu \mathrm{m}$ entrance window at the front (mirror) end. This is sufficient to prevent moisture (on the ground) and atomic oxygen (in orbit) from diffusing into the stick. Launch loads are estimated not to be an issue for the small cylinder. The Be housing, included in the Monte Carlo instrument simulations, has minimal impact on throughput. We are currently evaluating the merits of making the rear end of the scattering slab of Be rather than LiH. Mostly higher energy ( $>10$ $\mathrm{keV}$ ) photons reach the rear end. The higher density of Be $\left(1.85 \mathrm{~g} \mathrm{~cm}^{-3}\right)$ compared to that of $\mathrm{LiH}$ $\left(0.82 \mathrm{~g} \mathrm{~cm}^{-3}\right)$ leads to an increased fraction of high-energy photons Compton scattering in the rear end of the stick.

PolSTAR uses 17 flight-proven $32 \times 32$ pixel CZT hybrid detectors. Each pixel is attached to a readout circuit on a custom-designed low-noise application-specific integrated circuit (ASIC). NuSTAR has two focal plane modules, each constructed of a $2 \times 2$ array of detectors on wedgeshaped ceramic boards (Figure 7). PolSTAR packages the detectors slightly differently, with 16 detectors arranged in four $1 \times 4$ array mod-

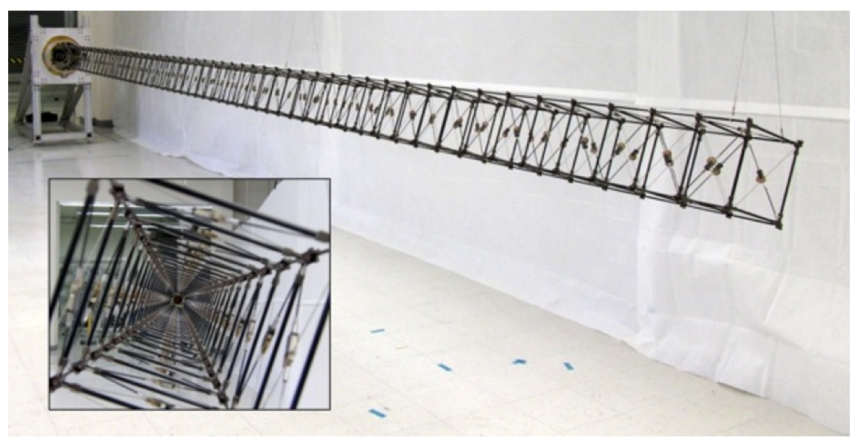

Figure 5: Essential to the PolSTAR and NuSTAR designs is a deployable mast which extends to 10.14 -m after launch. Using a deployable structure allowed NuSTAR and would allow PolSTAR to launch on a Pegasus XL rocket. This extendable mast was built by ATK Goleta, specializing in space-based deployable structures. These images are from a full deployment test of the NuSTAR flight mast at ATK Goleta in August 2009.

ules forming the box that surrounds the LiH stick (Figure 2). The final detector ("Detector 17") is located behind the $\mathrm{LiH}$ stick, perpendicular to the incident photon path as a tail catcher, enabling the imaging of the observed source with photons not interacting in the scattering element. PolSTAR uses these images to verify pointing throughout an observation.

Each pixel in the CZT detector has an independent discriminator, and individual X-ray photons trigger the readout process. On-board processors, one for each detector module, identify the row and column with the largest pulse height and read out pulse height information from this pixel as well as its eight neighbors, as on NuSTAR. Unlike CCDs, CZT detectors are non-integrating and self-triggering: for each each incident photon the charge deposited in the detectors is collected within $\leq 1 / 2 \mu$ s and is subsequently read out with an electronic processing time of $2.5 \mathrm{~ms}$ per event. The event time is recorded to an accuracy of $2 \mu$ s relative to the on-board clock and with an absolute timing accuracy of $\leq 2 \mathrm{~ms}$ limited by the stability of the spacecraft clock. The design replicates the timing capabilities of $N u S$ $T A R$ which surpass those of $\mathrm{X}$-ray telescopes with CCD detectors and have led to numerous discoveries (e.g. Mori et al., 2013; Bachetti et al., 2014, 


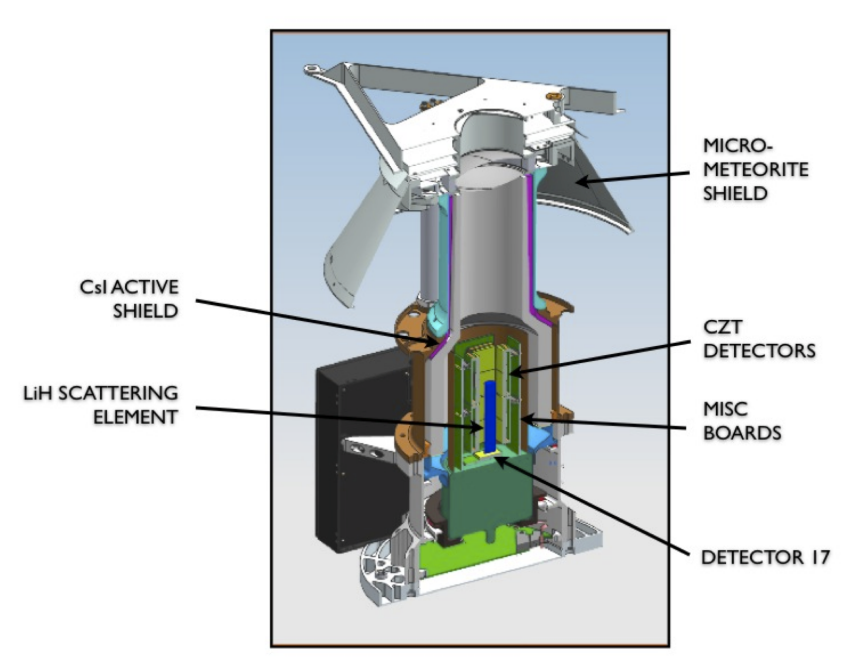

Figure 6: Design of the PolSTAR polarimeter showing the $\mathrm{LiH}$ scattering element surrounded by a CZT detector assembly inside a fully active CsI shield. Note to the copyeditor: please make this figure a factor of $\mathbf{1 . 5}$ larger.

2015; Rana et al., 2009). Additional details about the NUSTAR detectors and ASICs were described by Hailey et al. (2010b), Kitaguchi et al. (2010b), and Harrison et al. (2010b).

Metrology system: PolSTAR uses the NuS$T A R$ build-to-print metrology system, consisting of two infrared lasers mounted on the optics bench that focus beams on two corresponding detectors on the focal plane bench. The lasers spots are measured to an accuracy of $10 \mu \mathrm{m}\left(0.1^{\prime \prime}\right)$ and, combined with the instrument star camera data, track the thermal mast motions and enable accurate knowledge of the X-ray focal point position. The metrology system can be used to track the movement of the focal spot during the observations (Figure 8). Liebe et al. (2012) gives a detailed description of the system.

Shield module: PolSTAR's equatorial orbit, identical to that of NUSTAR, provides a low cosmic-ray flux and minimizes South Atlantic Anomaly (SAA) passage, thereby enabling re-use of the NuSTAR's low mass, cost-effective shield configuration. The polarimeter is contained inside a CsI active anti-coincidence shield with a photomultiplier tube, essentially identical to the one used on NuSTAR, but with an elongated geom-

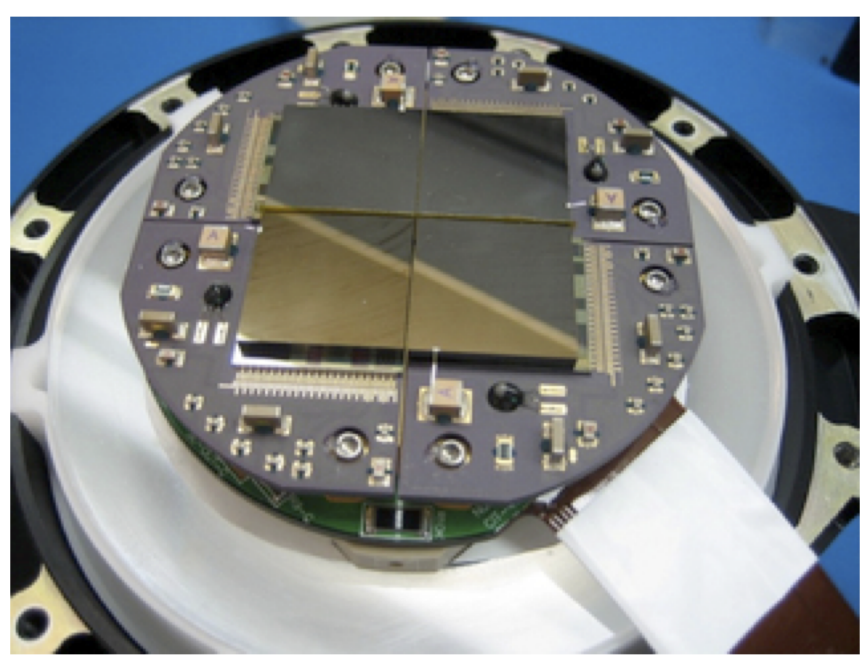

Figure 7: NuSTAR focal plane module, consisting of a $2 \times 2$ array of CZT detectors. PolSTAR uses identical detectors.

etry to accommodate the polarimeter. The rear portion of the CsI shield is $1.5 \mathrm{~cm}$ thick, and the side walls are $1.2 \mathrm{~cm}$ thick adjacent to the CZT detectors. The front (collimator) portion of the active shield has a wall thickness of $0.9 \mathrm{~cm}$ close to the CZT detectors tapering to $0.4 \mathrm{~cm}$ at the front side.

Calibration source: PolSTAR uses the same radioactive ${ }^{155} \mathrm{Eu}$ calibration source and deployable mounting as NuSTAR. The $10 \mu \mathrm{Ci}$ source is mounted on the side of the shield and can be moved into the field-of-view to monitor the gain and functionality of the detectors. When not deployed, the detectors are shielded from the source. As was done with NuSTAR, PolSTAR would use the calibration unit extensively during integration and testing, but only rarely on orbit.

\subsection{Mission and Mission Operations}

PolSTAR would be in a similar low-Earth $(\sim 530 \mathrm{~km}), 6^{\circ}$ inclination, near-circular orbit to NuSTAR. On orbit, PolSTAR has two operational modes. During solar-eclipse portions of orbits, PolSTAR would point at science targets and slowly rotate along the optical axis as a systematic error mitigation strategy. During the Sun-illuminated portions of orbits, the spacecraft would maintain the same pointing with respect to the science target, but would stop rotation. The 


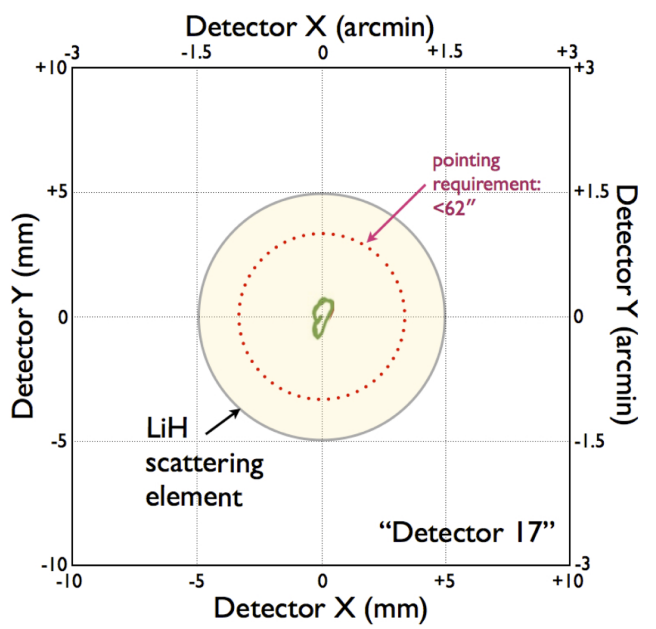

Figure 8: The NuSTAR focal spot moves as the spacecraft moves into and out of Earth's shadow. The green line compares the $N u S T A R$ focal spot for six orbits of the spacecraft to the size of the $\mathrm{LiH}$ scattering element and the $17^{\text {th }}$ CZT detector.

solar panel array can have a fixed attitude with respect to the Sun and charge the batteries. The typical eclipse rotation rate would be once per 10 min, or three full rotations per eclipse; for observing efficiency, the rotation rate would be tuned on a per-target basis to maintain an integer number of rotations per science observation. The settle time as the observatory goes in and out of eclipse is $<15$ sec. Note that this is a conservative approach, providing significant power margins for charging the batteries. NuSTAR nominally observes during both solar-illuminated and eclipse portions of orbits. We plan for a baseline mission with 18 months of science observations.

\subsection{Returned Data}

As per NuSTAR, for each event PolSTAR records the pulse height measured in the triggered CZT detector pixel and eight adjacent pixels, a time tag, and a bit indicating if the active CsI shield was triggered (54 bytes in total). Pol$S T A R$ can use NuSTAR algorithms to measure the energy of the photon striking the CZT detector based on the signal in the triggered and adjacent pixels. The processed PolSTAR data products include a list of events, and for each event the location of the energy deposition in the CZT detector, the sum $E_{\mathrm{i}}$ of all the energies recorded in the CZT detectors owing to one or multiple interactions of the photon with the detector material, the time of the event trigger, the offsets of the optical axis from the center of the scatterer in detector coordinates, and the roll angle of the spacecraft. The data products include the instrument response matrices, i.e., lookup tables giving the distribution of the observables as function of energy, polarization fraction and angle, the offsets and roll angle. The science products consist of the constraints on the model parameters, i.e., the parameters describing the flux, polarization fraction and polarization angle as function of energy. The constraints are derived from forward folding the model with the instrument response matrices and comparing the resulting distributions with the measured ones (see next section).

\section{Analysis Methods, Instrument Simula- tions, and Projected Performance}

\subsection{Analysis Methods}

The polarization properties of a quasimonochromatic electromagnetic wave can be described entirely by the four Stokes parameters (all having the units of intensity): the beam intensity $I$, the parameters $Q=p_{0} I \cos \left(2 \psi_{0}\right)$ and $U=p_{0} I \sin \left(2 \psi_{0}\right)$, describing linear polarization, where $p_{0}$ and $\psi_{0}$ are the polarization fraction and angle, and the circular polarization $V$ (Stokes, 1852). PolSTAR measures $I, Q$ and $U$ but is not sensitive to $V$. We use an analysis based on assigning Stokes parameters to each individual event (Kislat et al., 2015). The main advantage of the analysis described in the following over alternative methods (i.e. fitting the azimuthal scattering angle distribution with a suitable template) is (besides the ease of the involved calculations) that the Stokes parameters are normally distributed with a mean centered on 0 if the signal is unpolarized. The significance of a polarization detection and the confidence intervals on derived parameters (i.e. the polarization fraction and angle) thus follow from the well understood properties of Gaussian distributions. Assuming that the $i^{\text {th }}$ detected photon scattered along the optical axis, 
we use the position $x_{i}, y_{i}$ of the photon detection in the plane perpendicular to the optical axis to calculate the azimuthal scattering angle $\alpha_{i}$ measured relative to the celestial North direction. We then define

$$
\begin{aligned}
q_{i} & =\cos \left(2\left(\alpha_{i}-\frac{\pi}{2}\right)\right), \\
u_{i} & =\sin \left(2\left(\alpha_{i}-\frac{\pi}{2}\right)\right),
\end{aligned}
$$

where the terms $\frac{\pi}{2}$ account for the fact that photons scatter preferentially perpendicular to the polarization direction. As the Stokes parameters are additive, the Stokes parameters of the signal are simply the sum of the $q_{i}$ and $u_{i}$ of all $N$ observed events which pass the analysis cuts (i.e. which were not flagged as background events which triggered the CsI shield). We define the reduced Stokes parameters:

$$
\begin{aligned}
& \mathcal{Q}_{r}=\frac{2}{\mu N} \sum_{i=1}^{N} q_{i}, \\
& \mathcal{U}_{r}=\frac{2}{\mu N} \sum_{i=1}^{N} u_{i}
\end{aligned}
$$

with $\mu$ being the modulation factor, i.e. the fractional amplitude of the azimuthal scattering angle distribution for a $100 \%$ linearly polarized X-ray beam. The measured polarization fraction and angle are then given by

$$
\begin{aligned}
p & =\sqrt{\mathcal{Q}_{r}^{2}+\mathcal{U}_{r}^{2}}, \\
\psi & =\frac{1}{2} \arctan \frac{\mathcal{U}_{r}}{\mathcal{Q}_{r}} .
\end{aligned}
$$

The Stokes parameters follow a Gaussian distribution, and the one sigma measurement error is given by (Kislat et al., 2015):

$$
\begin{aligned}
\sigma\left(\mathcal{Q}_{r}\right) & =\sqrt{\frac{1}{N-1}\left(\frac{2}{\mu^{2}}-\mathcal{Q}_{r}^{2}\right)}, \text { and } \\
\sigma\left(\mathcal{U}_{r}\right) & =\sqrt{\frac{1}{N-1}\left(\frac{2}{\mu^{2}}-\mathcal{U}_{r}^{2}\right)} .
\end{aligned}
$$

The polarization fraction is restricted to values $p \geq 0$, with the probability distribution of the measurement $p$ given the true polarization fraction $p_{0}$ (Vinokur, 1965; Weisskopf et al., 2006; Krawczynski, 2011; Kislat et al., 2015):

$$
P\left(p \mid p_{0}\right)=\frac{N p \mu^{2}}{2} e^{-\frac{N \mu^{2}}{4}\left(p^{2}+p_{0}^{2}\right)} I_{0}\left(\frac{N \mu^{2} p p_{0}}{2}\right),
$$

where $I_{0}$ is the modified Bessel function of order zero. We use Equation 6 to estimate the measurement errors on the polarization fractions measured with PolSTAR . For this purpose,we numerically integrate Eq. (6) and find the range $\left[p_{1}, p_{2}\right]$ within which $67 \%$ of measurements are expected, such that $P\left(p_{1} \mid p_{0}\right)=P\left(p_{2} \mid p_{0}\right)$.

The polarization angle is described by a normal distribution with

$$
\sigma(\psi) \approx \frac{1}{p \mu \sqrt{2(N-1)}} .
$$

The minimum detectable polarization (MDP) is defined as the $99 \%$ confidence level upper limit found for an unpolarized source (Weisskopf et al., 2006; Krawczynski, 2011; Kislat et al., 2015),

$$
\operatorname{MDP} \approx \frac{4.29}{\mu \sqrt{N}}
$$

\subsection{Measurements in the presence of backgrounds}

PolSTAR intersperses science observations with slightly offset $\left(<1^{\circ}\right)$ observations to measure the local background (rate of events not initiated by the source, e.g. by cosmic rays and photons from the cosmic X-ray background), with offset pointing durations tailored on a per-target basis. The background regions would be chosen to avoid bright X-ray sources in the field of view.

For all but the brightest sources, $\mathcal{Q}_{r}$ and $\mathcal{U}_{r}$ are then measured independently for on-source and off-source observations. The Stokes parameters of the source are then

$$
\begin{aligned}
& \mathcal{Q}_{\text {source }}=\mathcal{Q}_{\text {on }}-w_{\text {off }} \mathcal{Q}_{\text {off }}, \\
& \mathcal{U}_{\text {source }}=\mathcal{U}_{\text {on }}-w_{\text {off }} \mathcal{U}_{\text {off }},
\end{aligned}
$$

where the weight $w_{\text {off }}$ is the ratio of the on-source observation time divided by the off-source observation time:

$$
w_{\mathrm{off}}=\frac{t_{\mathrm{on}}}{t_{\mathrm{off}}} .
$$


The optimal choice of $w_{\text {off }}$ depends on the expected signal rate $R_{\mathrm{S}}$ and background rate $R_{\mathrm{BG}}$ for a given source. By minimizing the expected uncertainties on $\mathcal{Q}_{\text {source }}$ and $\mathcal{U}_{\text {source }}$ from Equation (5), one finds the optimum value (Kislat et al., 2015)

$$
w_{\mathrm{off}}=\sqrt{1+R_{\mathrm{S} / \mathrm{B}}},
$$

with $R_{\mathrm{S} / \mathrm{B}}=R_{S} / R_{B G}$. Accounting for the statistical errors on the Stokes parameters of the signal and the background, the MDP becomes

$$
\mathrm{MDP}=\frac{4.29 \sqrt{R_{S}+R_{B G}}}{\mu \sqrt{T}\left(R_{S}+R_{B G}-\sqrt{R_{B G}\left(R_{B G}+R_{S}\right)}\right)}
$$

with $T=t_{\text {on }}+t_{\text {off }}$ being the total on-source and background observation time. Based on the worst-case estimate of the PolSTAR background, PolSTAR would spend $\sim 15 \%$ of the observation time of the baseline mission on background observations.

An offset of the focal point from the center of the scattering element leads to an asymmetry in the azimuthal scattering distribution owing to the direction dependent absorption in the scattering element and a geometrical bias from folding the mirror PSF with the cross section of the scattering element (Beilicke et al., 2014b). The full Pol$S T A R$ data analysis corrects for pointing offsets with the help of a forward-folding analysis. The latter uses a template library of simulated events generated according to a $E^{-1}$ power-law spectrum for a matrix of focal spot offsets with Stokes parameters $Q, U= \pm 1$. A particular observation is modeled by drawing events from the library to mimic the pointing history during the observation. Events with certain $Q$ and $U$ values are drawn to simulate a beam with certain net Stokes $Q$ and $U$ values, and are weighted according to the assumed energy spectrum. A chi-square minimization is then used to find the best-fit model parameters and to derive model uncertainties. The details of this analysis are the subject of a future paper.

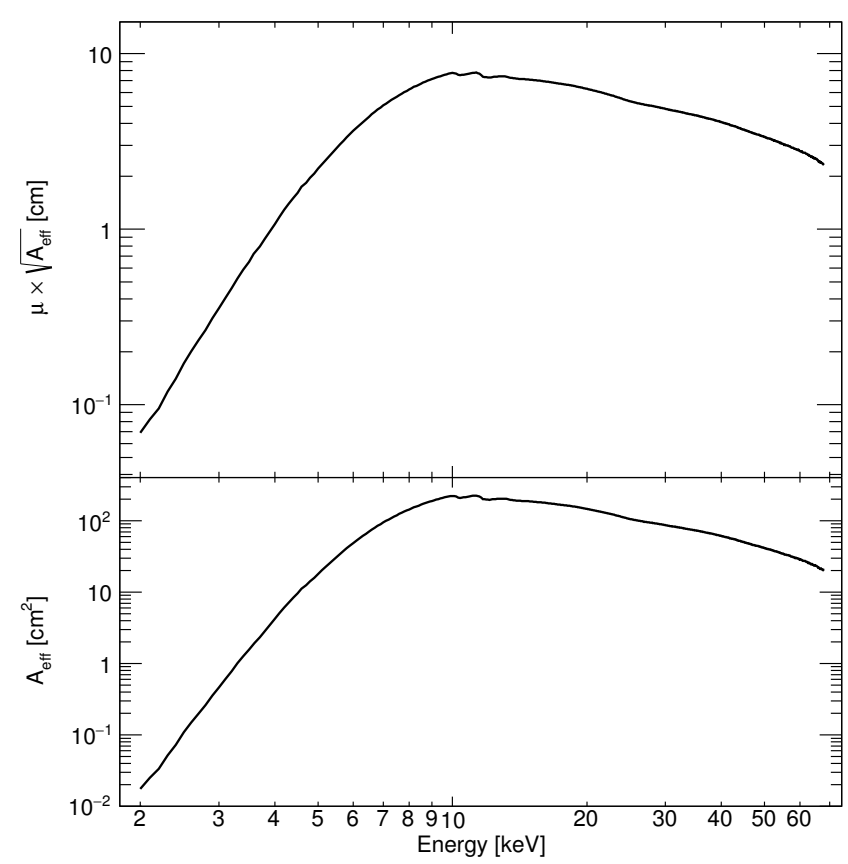

Figure 9: The upper panel shows PolSTAR's modulation factor $\mu$ times the square root of the effective detection area $A_{\text {eff }}$ as function of energy, and the lower panel shows $A_{\text {eff }}$ as function of energy. For comparison, OSO-8 had a peak continuum radiation effective area of $0.6 \mathrm{~cm}^{2}$ at $9 \mathrm{keV}$ (Kestenbaum et al., 1976). A Crab-like source leads to a flux density rate $>1$ count $\mathrm{sec}^{-1} \mathrm{keV}^{-1}$ over the entire range $3.5-25 \mathrm{keV}$.

\subsection{PolSTAR Instrument Simulations and Per-} formance

We estimate the PolSTAR performance by combining $N u S T A R$ pre-flight and in-orbit results with Monte Carlo simulations of the polarimeter response. The simulations use the GEANT-4 simulation package with the Livermore Low-Energy Electromagnetic Models physics list (Agostinelli et al., 2003). The simulations use the results from a ray-tracing code developed for $N u S T A R$ and include the $N u S T A R$-measured energy-dependent quantum efficiency of the CZT detectors. The latter include the effects of photon absorption by the cathode and the inactive transition layer between the cathode and the CZT. The simulations account for the possibility of multiple interactions of the high-energy photons within the scatterer and the detectors.

Figure 9 presents the total effective area $A_{\text {eff }}$ of PolSTAR (including the optics, scatterer, and detector efficiencies) as a function of energy as 


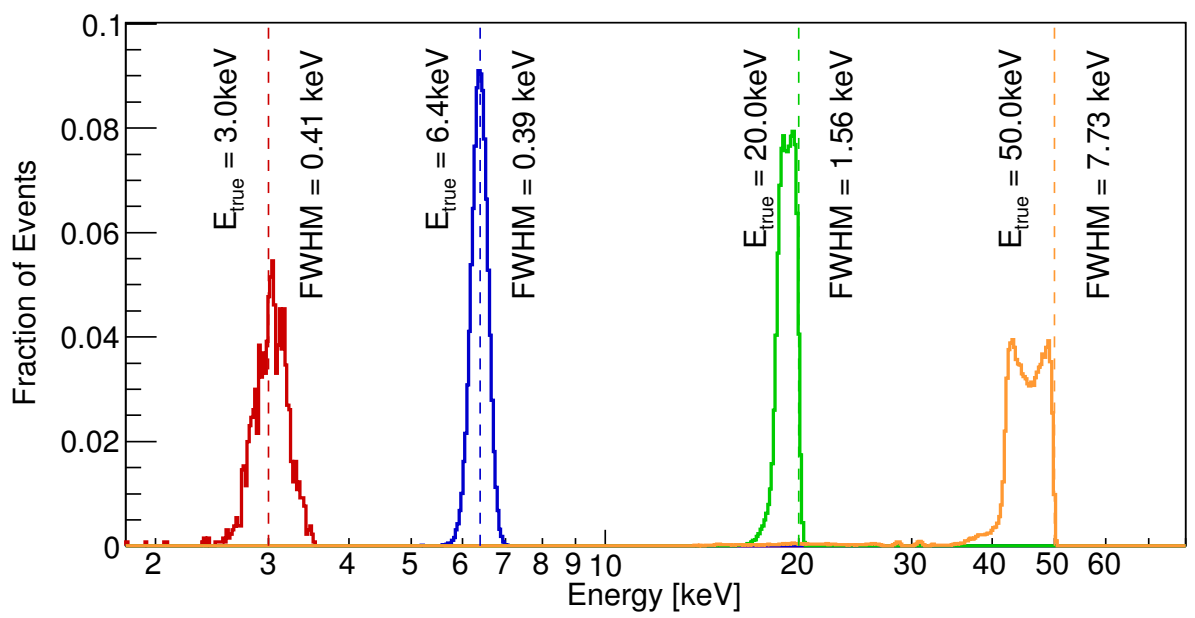

Figure 10: Energies deposited in the CZT detectors for different incident photon energies.

well the product of the modulation factor $\mu$ times the square root of the effective area. The latter product can be used as figure of merit characterizing the differential sensitivity of a polarimeter as the minimum detectable polarization fraction follows the scaling law MDP $\propto\left(\mu \sqrt{A_{\text {eff }}}\right)^{-1}$. For a source with a Crab like flux and energy spectrum the $3-15 \mathrm{keV}$ detection rate is $R_{\mathrm{src}}=108 \mathrm{~Hz}$ (CBE, $97 \mathrm{~Hz}$ requirement). The modulation factor $\mu$ gives the amplitude of the sinusoidal modulation of the azimuthal scattering angle distribution for a $100 \%$ polarized signal. For PolSTAR, the simulations give $\mu \approx 0.52$ largely independent of energy.

PolSTAR's energy resolution is limited by the CZT detector/readout resolution to about 0.4 keV FWHM at low $(<10 \mathrm{keV})$ energies. At higher energies $(>10 \mathrm{keV})$ an increasing fraction of the primary photon's energy is given to the Compton electron. Figure 10 shows the detector response for a few exemplary incident photon energies. After re-normalizing the energies deposited in the CZT detectors to the incident photon energy, we infer energy resolutions of $0.4 \mathrm{keV} \mathrm{FWHM}$ at $<10$ $\mathrm{keV}, 1.65 \mathrm{keV}$ FWHM at $20 \mathrm{keV}$ and $8.5 \mathrm{keV}$ FWHM at $50 \mathrm{keV}$. The full (forward folding) analysis makes use of the fact that each ring of pixels surrounding the scattering element sees photons preferentially from the front-end of the scattering element with an energy dependent exponen-

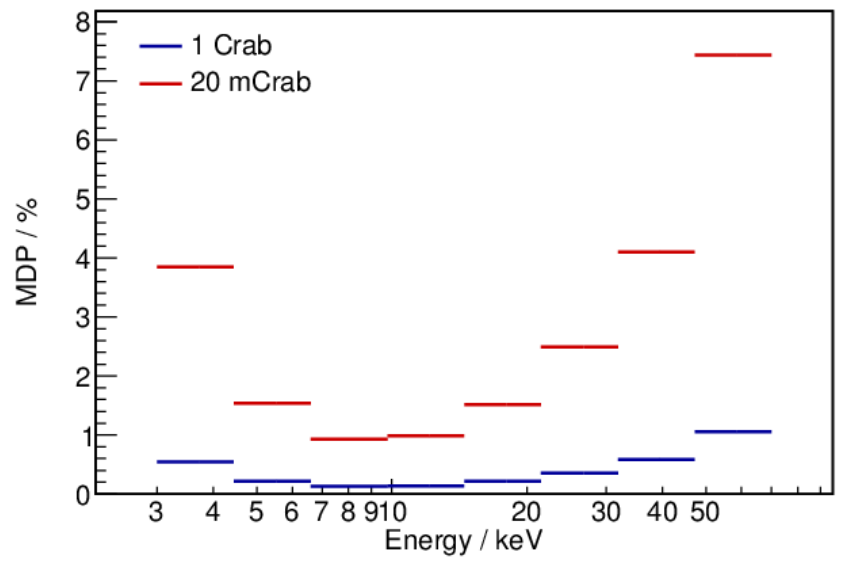

Figure 11: PolSTAR's polarization sensitivity for dim (upper red bars) and bright (lower blue bars) sources. The lines show the Minimum Detectable Polarization fraction (MDP, 99\% CL) as a function of source flux in eight statistically independent energy bins assuming $860 \mathrm{ksec}(10$ days) of on-source exposure time for a source with a Crablike spectrum.

tially suppressed distribution of scattering locations deeper into the element. Taking into account where the photons strike the CZT detector assembly, the energy resolutions can be improved for a subset of the events. For example, the energy of $50 \mathrm{keV}$ events detected at the front end of the second detector ring (counted from the front end) can be reconstructed with an effective energy resolution of $4 \mathrm{keV}$ FWHM.

For dim sources, PolSTAR's sensitivity de- 


\begin{tabular}{|c|c|c|c|}
\hline Effect & \multicolumn{2}{|c|}{$\begin{array}{l}\text { Pol. fraction error for non-rotating } \\
\text { instrument[\%] }\end{array}$} & \multirow[t]{2}{*}{$\begin{array}{l}\text { Error suppressed } \\
\text { by rotation? }\end{array}$} \\
\hline Energy range (keV): & $3-15$ & $15-50$ & \\
\hline $\begin{array}{l}\text { Detector and background inhomo- } \\
\text { geneities }\end{array}$ & 0.25 & 0.25 & $\mathrm{Y}$ \\
\hline LiH mechanical tolerances $(0.2 \mathrm{~mm})$ & 0.04 & 0.13 & $\mathrm{Y}$ \\
\hline $\begin{array}{l}\text { PSF unc. (pre-/post-launch } N u S- \\
\text { TAR comparison) }\end{array}$ & 0.28 & 0.24 & $\mathrm{Y}$ \\
\hline $\begin{array}{l}0.1 \mathrm{keV} \text { energy calibration error, } \\
\text { limiting pointing offset corrections }\end{array}$ & 0.1 & 0.001 & $\mathrm{Y}$ \\
\hline Pointing knowledge error & $1.4 / 0.25^{\mathrm{a}}$ & $1.3 / 0.1^{\mathrm{a}}$ & $\mathrm{N}$ \\
\hline
\end{tabular}

Table 4: PolSTAR worst-case systematic errors $(99.7 \% \mathrm{CL})$ for a source consistently offset by $3 \mathrm{~mm}\left(62^{\prime \prime}\right)$ from the center of the field of view.

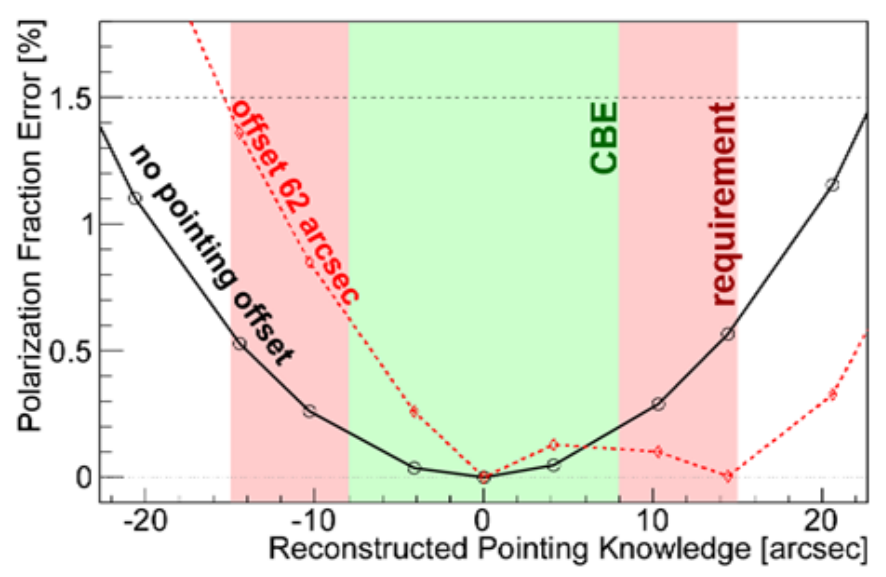

Figure 12: Monte Carlo simulation of the systematic 3$15 \mathrm{keV}$ polarization fraction error as a function of the pointing knowledge error. For a nominal position centered on the $\mathrm{LiH}$ stick (black circles), a $15^{\prime \prime}\left(8^{\prime \prime}\right)$ positional uncertainty translates into a systematic polarization fraction uncertainty of $0.6 \%(0.2 \%)$. For a nominal position centered $62^{\prime \prime}$ from the LiH stick center (red triangles), a $15^{\prime \prime}$ positional uncertainty can produce polarization fraction errors as high as $1.4 \%$. All systematic uncertainties quoted at the $99.7 \%$ CL.

pends on the level of background counts. We conservatively assume the background per detector measured in-orbit with the NuSTAR CZT detectors (Wik et al. 2014). For NuSTAR stray cosmic X-ray background light leaking through the aperture stop dominates the background below $\sim 20 \mathrm{keV}$. Internal radiation activated by the orbital environment dominates above $20 \mathrm{keV}$. The PolSTAR background should be substan- tially lower than the $N u S T A R$ background as the detectors do not see the sky directly but are oriented towards the scattering slab, and the latter absorbs a considerable fraction of the events at $\leq 10 \mathrm{keV}$ energies. The NuSTAR-based estimate predicts a worst-case 5-20 keV background rate of $R_{\mathrm{bkg}}=0.94 \mathrm{~Hz}$. Simulations are underway to improve this estimate. Bright sources within $5^{\circ}$ of a target cause additional stray light issues for $\mathrm{NuS}$ $T A R$ which is only a concern for five targets in the PolSTAR baseline observation program ( $(4)$. This can be mitigated through a combination of modeling, off-target measurements, and data censoring. We are also evaluating the merits of incorporating a flight-ready stray light baffle that was built too late for $N u S T A R$ to be incorporated.

The PolSTAR sensitivity is best between 5-15 $\mathrm{keV}$ (see Figure 11). The sensitivity decreases at lower energies owing to the limited scattering efficiency in the scattering element. At higher energies, the assumed steep energy spectrum of the astrophysical source and the declining mirror effective area limit the sensitivity.

We used simulated data sets to estimate systematic errors. The relevant figure of merit is the spurious polarization measured for an unpolarized X-ray beam. Table 4 lists the main sources of systematic errors before accounting for their suppression through the spacecraft roll. The largest error stems from the practical limitations of flatfielding the detectors. Note that the spacecraft 


\begin{tabular}{|l|c|c|c|}
\hline & $\begin{array}{l}\text { PolSTAR Req't } \\
(99.7 \% \mathrm{CL})\end{array}$ & $\begin{array}{c}\text { PolSTAR CBE } \\
(99.7 \% \mathrm{CL})\end{array}$ & $\begin{array}{l}\text { NuSTAR } \\
\text { Actuals }\end{array}$ \\
\hline Control & $\leq 40^{\prime \prime}$ & $11.3^{\prime \prime}$ & $8^{\prime \prime}$ \\
\hline Stability - science orbit & $\leq 40^{\prime \prime}$ & $12.9^{\prime \prime}$ & $11^{\prime \prime}$ \\
\hline Combined & $\leq 62^{\prime \prime}$ & $17.1^{\prime \prime}$ & $13.6^{\prime \prime}$ \\
\hline \hline
\end{tabular}

Table 5: PolSTAR pointing requirements.

roll suppresses most systematic errors by averaging over detector non-uniformities and spacecraft asymmetries. The main contribution to the residual systematic error is expected to come from the pointing knowledge error which varies as the spacecraft rolls. Figure 12 presents the resulting spurious polarization for a target both centered on-axis and offset by $3 \mathrm{~mm}\left(62^{\prime \prime}\right)$. Based on these simulations, we find that in order to meet the requirement that systematic polarization fraction errors be $\leq 1.5 \%$, we are required to maintain the target within $62^{\prime \prime}$ of the $\mathrm{LiH}$ stick center and have reconstructed pointing knowledge $\leq 15^{\prime \prime}$, where all numbers are at the $99.7 \%$ CL. Table 5 presents the budgeted requirements on the pointing system derived from the science constraints on the systematic errors and compares them to the Pol$S T A R$ CBE and the NuSTAR actuals. By requiring pointing control $\leq 40^{\prime \prime}$ (from the spacecraft) and pointing stability $\leq 40^{\prime \prime}$ (from the combined motions of the spacecraft and mast), we can maintain the target within $62^{\prime \prime}$ of the desired location aligned with the $\mathrm{LiH}$ stick center (Root of Sum of Squares $(\mathrm{RSS})=57^{\prime \prime}$; CBE is $\left.17^{\prime \prime}\right)$. Using the metrology and star tracker data on the ground, NuSTAR achieves reconstructed pointing knowledge to an accuracy of $8^{\prime \prime}(99.7 \%$ CL). The rotation of PolSTAR is sufficiently slow that this performance is not degraded.

\section{Science Investigations}

We designed PolSTAR for a mission duration of 18 months. In this time, PolSTAR can observe the 24 sources listed in Table 6 . The sources include the brightest and best-studied sources of several source classes as well as two targets of opportunity. The PolSTAR observations can be used for physics-type experiments, validating or falsifying the leading paradigms of where and how the X-ray emission originates in these sources. The science objectives can be summarized as follows:

Objective 1: Reveal black hole accretion flows: PolSTAR combines spectroscopic, timing, and polarimetric information to map the innermost accretion flows around stellar mass and supermassive black holes, where gravitational potential energy is converted into radiation and mechanical energy. PolSTAR's 3-D information about the structure of the accretion flow tests theories of black hole accretion (Dovčiak et al., 2004, 2008; Li et al., 2009; Schnittman and Krolik, 2009, 2010; Dovčiak et al., 2011). PolSTAR's energy band is ideally suited to decomposing the spectra of accreting objects into components from accretion disks (3-8 keV; only in the case of stellar mass black holes), hot coronal regions (3-50 keV), coronal emission reflected and re-processed off the accretion disk (6-50 keV, including the $>10 \mathrm{keV}$ Compton hump), and, in some cases jets (which in some scenarios may contribute significantly above $\sim 40 \mathrm{keV}$ ). Einstein's theory of General Relativity (GR) makes as-yet untested predictions about the behavior of matter and radiation in the extremely curved and twisted spacetime around black holes. PolSTAR can search for the predicted signatures of the general relativistic Lense-Thirring precession and Bardeen-Peterson warp of the inner accretion flow in the strong gravity regime.

Objective 2: Reveal the magnetic backbone of blazar jets: PolSTAR can test leading theories of how actively accreting supermassive black holes form, accelerate, and collimate powerful outflows (jets) by accurately measuring the time evolution of the polarization angle of the X-ray emission from blazars (supermassive black holes with jets 


\begin{tabular}{|l|l|l|l|l|l|}
\hline Source & Source Type & $\begin{array}{l}\text { Flux } \begin{array}{r}2- \\
12 \\
\text { keV } \\
{[\mathrm{mCrab}]}\end{array} \\
\end{array}$ & $\begin{array}{l}\text { On-source } \\
\text { time } \\
{[\text { days }]}\end{array}$ & $\begin{array}{l}\text { MDP } \\
3-15 \mathrm{keV}] \\
99 \% \mathrm{CL}\end{array}$ & $\begin{array}{l}\text { Pol. } \\
\text { Fraction } \\
\text { Error [\%] } \\
3-15 \mathrm{keV}\end{array}$ \\
\hline \hline Cyg X-1 & Stellar BH & 414 & 3.4 & 0.2 & 0.09 \\
GRS 1915+105 & Stellar BH & 717 & 15.2 & 0.1 & 0.03 \\
LMC X-3 & Stellar BH & 20.1 & 13.3 & 0.7 & 0.35 \\
Cyg X-3 & Stellar BH & 168 & 0.9 & 0.7 & 0.30 \\
Flaring source & Stellar BH & 82.8 & 2.1 & 0.7 & 0.31 \\
\hline NGC 4151 & Supermass. BH & 5.9 & 5.5 & 2.6 & 1.51 \\
MCG-5-23-16 & Supermass. BH & 4.4 & 6.3 & 3.0 & 1.84 \\
MCG-6-30-15 & Supermass. BH & 3.9 & 7.8 & 3.0 & 1.85 \\
\hline Mrk 421 & HSP Blazar & 14 & 5.7 & 1.3 & 0.71 \\
Mrk 501 & HSP Blazar & 5.1 & 10.0 & 2.1 & 1.28 \\
3C 273 & FSRQ & 4.9 & 1.9 & 5.1 & 3.05 \\
PKS 1510-08 & FSRQ & 2.57 & 5.9 & 5.0 & 3.13 \\
Flaring Blazar & Blazar & 10 & 0.5 & 7.2 & 4.30 \\
\hline 1E 2259+586 & AXP & 10.2 & 9.3 & 1.3 & 0.72 \\
4U 0142+61 & AXP & 4.8 & 10.1 & 2.3 & 1.35 \\
SGR 1806-20 & SGR & 4.5 & 11.2 & 2.2 & 1.35 \\
\hline Vela X-1 & Acc. Pulsar & 48.3 & 0.7 & 1.8 & 0.81 \\
GX 301-2 & Acc. Pulsar & 22 & 1.9 & 1.7 & 0.86 \\
Her X-1 & Acc. Pulsar & 13.5 & 3.8 & 1.6 & 0.89 \\
Sco X-1 & Acc. NS & 1173 & 0.1 & 1.0 & 0.35 \\
4U 1700-377 & Acc. NS & 50.7 & 0.6 & 1.8 & 0.81 \\
Cyg X-2 & Acc. NS & 50 & 0.6 & 1.8 & 0.81 \\
Crab & Rot. Pulsar & 1000 & 0.1 & 1.0 & 0.35 \\
Vela & Rot. Pulsar & 7.9 & 2.2 & 3.2 & 1.85 \\
\hline
\end{tabular}

Table 6: The 24 targets of the baseline PolSTAR mission. Tabulated minimum detectable polarization fraction (MDP) and polarization fraction errors represent the statistical uncertainties for mission requirements.

pointing at us). The theories invoke a helical magnetic field moving through the X-ray emission region, and predict smooth swings of the polarization angle of the synchrotron continuum emission over time (Marscher et al., 2008). Polarization angle swings are predicted to be more pronounced in the $\mathrm{X}$-ray band than in the radio or optical bands because the X-ray emitting regions are smaller and more uniform, as evidenced by their fast, large amplitude flares (Krawczynski et al., 2013).

Objective 3: Explore the new physics of strongly magnetized neutron stars: PolSTAR observations of anomalous X-ray pulsars (AXPs) and soft gamma-ray repeaters (SGRs) test the magnetar hypothesis, which posits that the high- energy emission from these objects is driven by extremely strong $\left(10^{14}-10^{15} \mathrm{G}\right)$ neutron star magnetic fields. The magnetar model predicts extremely high ( 20-100\%) polarization fractions in the PolSTAR energy band (e.g. Shaviv et al., 1999; Fernández and Davis, 2011; Taverna et al., 2014). Phase-resolved polarization measurements (i.e., folding the polarization data with the rotation period of the neutron star) can constrain the magnetic field configuration in the magnetosphere, distinguishing a pure dipole field from a twisted magnetic field (Fernández and Davis, 2011). PolSTAR observations of magnetars and strongly magnetized accreting neutron stars can afford, for the first time, the capability to detect vacuum birefringence, a prediction of quantum 
electrodynamics (QED) in ultra-strong magnetic fields that cannot be tested in terrestrial laboratories (Kii, 1987; Meszaros et al., 1988; Taverna et al., 2014). Again, PolSTAR's broad energy band pass is key for this study as the effects manifest themselves in the 5-50 keV energy band. In accreting pulsars with magnetic field strengths of $\sim 10^{12} \mathrm{G}$, this band covers the vacuum resonance (where plasma and vacuum birefringence compete) as well as the cyclotron line energy (Kii, 1987; Mészáros, 1992; Krawczynski et al., 2011; Ghosh et al., 2013).

In the following, we discuss the observations for each of the three objectives.

\subsection{Dissect the Black Hole Accretion Flows onto Stellar Mass Black Holes}

PolSTAR Can Measures the Polarization Properties of Multiple Emission Components

The observation plan includes five bright stellar mass black holes in Galactic X-ray binaries with particularly deep observations of the bright systems Cyg X-1 and GRS 1915+105. PolSTAR can measure polarization fractions with statistical accuracies of $0.5 \%(1 \sigma)$ in as many as 40 (Cyg X1) to 320 (GRS $1915+105)$ independent temporal and energy bins. The high signal-to-noise data sets sample the polarization properties as functions of time, flux, and emission state. Figure 13 (left) shows a simulated three-day observation of Cyg X-1 in the soft state highlighting the quality of the data with detailed information about the polarization properties of the thermal disk emission and the direct and reflected coronal emission. Observing the source in different states can disentangle the polarization of the individual emission components.

\section{Studies of Black Hole Coronas}

Spectroscopic observations of black holes require the presence of a hot plasma to explain the power law spectral component dominating the emission at higher energies as Comptonized accretion disk photons (see e.g. Sunyaev and Thorne, 1973; Thorne and Price, 1975; Shapiro et al., 1976; Katz, 1976; Sunyaev and Titarchuk, 1980). Although coronas have been the subject of intense studies, their shapes, origin, and the roles they play in accretion systems are still a matter of debate (e.g. Zhang, 2013; Gilfanov and Merloni, 2014). X-ray polarimetry offers additional information that can be used to constrain the corona properties. Figure 13 (right) shows the simulated results of a three-day observation of GRS $1915+105$ in the power law state. The polarization properties of the two corona models differ markedly. PolSTAR's results enable using the corona as a diagnostic tool to understand the processes driving black hole accretion and jet formation.

Measurement of Black Hole Spin and the Orientation of the Inner Accretion Disk

Figure 14 shows that a seven-day exposure of GRS $1915+105$ in the thermal state promises to measure its black hole spin and inner accretion disk orientation. We performed a quantitative analysis of how well PolSTAR can measure the spin by generating a library of fitting templates derived from modeling the thermal emission from an optically thick, geometrically thin accretion disk for an array of black hole spin values. The modeling assumes the standard general relativistic Novikov-Thorne emissivity profile, and traces photons emanating from the accretion disk forward in time (see Krawczynski, 2012, for a detailed description of the general relativistic ray tracing code). The initial polarization and the polarization change associated with photon scattering off the accretion disk are modeled with the classical Chandrasekhar equations giving the Stokes parameters for the emission and reflection by a optically thick atmosphere (Chandrasekhar, 1960). The fitting templates are generated by folding the simulated Stokes parameter energy spectra with the detector response. After simulating one observed PolSTAR data set, a least squares fit is performed to determine the best-fit black hole parameters and the associated errors. The least squares analysis (see the right panel of Fig. 14) recovers the input dimension-less spin parameter and inclination and position angle of the inner accretion disk with $1 \sigma$ (combined statistical and systematic) accuracies of 0.02 (spin) and 

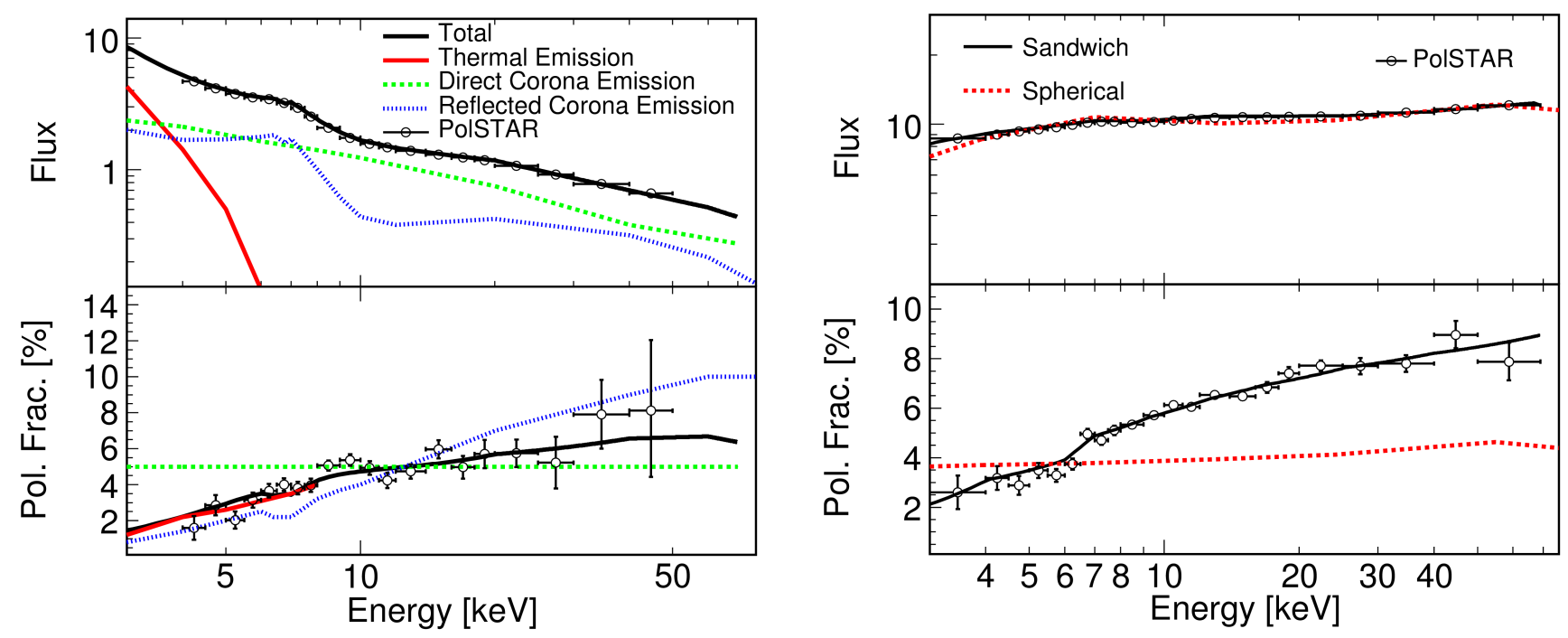

Figure 13: The broad energy range of PolSTAR covers all primary X-ray emission components of accreting compact objects, and provides a wide lever arm for distinguishing models. Left: Simulated 3-day observation of the black hole Cyg X-1 in the soft state, illustrating the distinct spectral and spectropolarimetric properties of the three primary emission components of accreting stellar mass black holes: thermal disk emission, direct coronal emission, and reflected coronal emission. The simulated data set (data points) assumed the model predictions for the total emission (solid line). (energy spectrum from Tomsick et al., 2014; polarization fractions and angles roughly consistent with the simulation results of Schnittman and Krolik, 2009, 2010 for a 10 solar mass black hole with a spin of $a=0.9$ accreting at $10 \%$ of the Eddington luminosity as seen at an inclination of $i=75^{\circ}$ ). Right: Simulated three-day observation of the black hole GRS 1915+105 in the power law state, showing that PolSTAR can distinguish between the modeled sandwich and spherical corona models. The simulated data set (data points) assumed the model predictions for the sandwich corona (solid line). The polarization fractions and angles are from the simulations of a 10 solar mass black hole with a spin of $a=0.9$, accreting at $10 \%$ of the Eddington luminosity as seen at an inclination of $i=75^{\circ}$ (Schnittman and Krolik, 2010, Fig. 3). In both figures, the upper flux panels refer to energy flux per logarithmic energy interval in units of $10^{-9} \mathrm{erg} \mathrm{cm}^{-2} \mathrm{~s}^{-1}$.

$\sim 2-3^{\circ}$ (inclination and position angle). The measurement errors for such a bright source are dominated by the systematic uncertainties (if we neglect astrophysical uncertainties); achieving measurements at this accuracy drive the systematic error requirements for PolSTAR. Spin measurements based on modeling the thermal X-ray continuum assume that the inner accretion disk is aligned with the binary orbit and that the disk emission from within the innermost stable circular orbit is negligible (e.g. see Gou et al., 2011, 2014, and references therein). Measurements based on modeling the iron line and reflection component rely on a number of assumptions regarding the geometry of the corona, the illumination profile, and the thermo-dynamic state of the reflecting disk material (e.g. see Tomsick et al., 2014, and references therein). PolSTAR can provide independent checks of these assumptions.

The orientation of the inner accretion disk can be compared to the orientation of the binary orbit and jet, if present (Figure 15). A misalignment between the inner accretion disk and the orbital plane would provide strong evidence for the general relativistic Bardeen-Peterson effect, while a non-zero angle between the spin axis of the inner disk and the jet would suggest mechanisms to bend the jet away from its original direction.

\section{Testing General Relativity's Prediction of Lense- Thirring Precession}

Low frequency quasi-periodic oscillations (hereafter QPOs) are regularly observed in the Xray light curves of accreting black holes (e.g. Remillard and McClintock, 2006, and references 

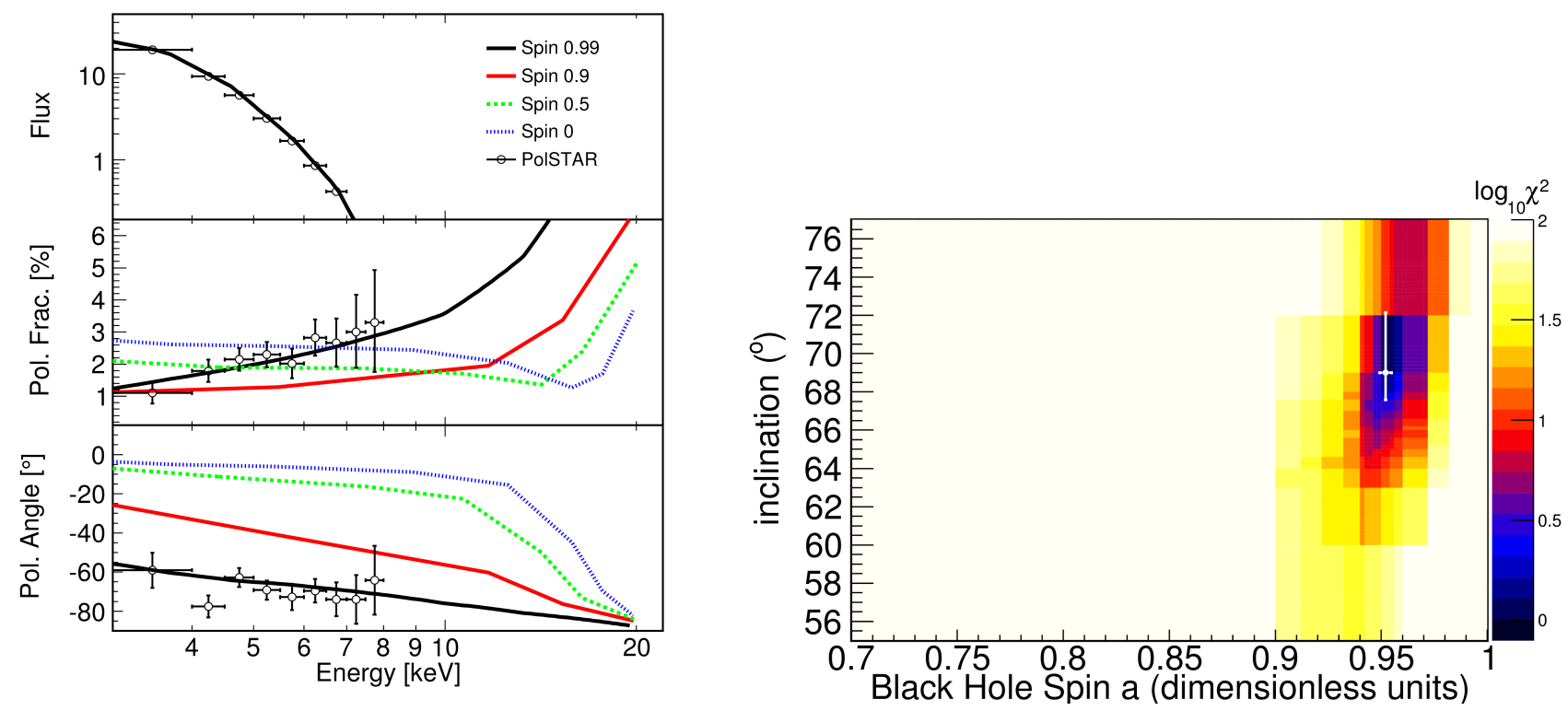

Figure 14: PolSTAR can provide an independent, geometric measure of black hole spin. Left: Simulated seven-day observation of GRS 1915+105 in the thermal state. We used the measured energy spectrum of (Ueda et al., 2010). The lines show the polarization fractions and angles from the simulations of a 10 solar mass black hole accreting at $10 \%$ of the Eddington luminosity for different values of the black hole spin as seen at an inclination of $i=75^{\circ}$ (from Schnittman and Krolik, 2009, Fig. 7). The simulated data points assume the polarization properties for the model with spin $a=0.99$ shown by the solid line. The flux is given in the same units as in Figure 13. Right: results of a quantitative analysis of simulated PolSTAR data, using a least squares fit to determine the black hole spin, inclination, and the orientation of the spin axis in the sky (not shown). The white dot shows the best-fit value of the $\chi^{2}$ fit at $a=0.952$, inclination $=69^{\circ}$ (input values: $a=0.95$ and inclination $=66^{\circ}$ ). The white cross shows the combined $1 \sigma$ statistical and systematic errors, which are primarily systematic for this bright source.

therein). The QPO and power spectral break frequencies are correlated in black hole X-ray binaries (Wijnands and van der Klis, 1999), and the QPO amplitude depends on system inclination (Motta et al., 2015; Heil et al., 2015). Both of these properties suggest that the QPOs are geometric in origin independent of any specific model. Broadband X-ray polarization probes geometry, and so can test the nature of black hole QPOs.

Perhaps the most successful model to explain QPOs considers precession of the inner accretion flow due to the relativistic effect of frame dragging. A spinning black hole twists up the surrounding space-time, inducing Lense-Thirring precession in test mass orbits. The model of Ingram et al. (2009) assumes that the entire inner accretion flow $\left(r<20 r_{\mathrm{g}}\right)$ precesses as a solid body, motivated by the simulations of Fragile et al. (2007). If this is indeed the true QPO mechanism, the X-ray polarization signature from black holes should also contain a QPO.
PolSTAR's broadband sensitivity is ideally suited for this kind of study. The precession leads to quasi-periodic large-amplitude changes of the polarization of the Comptonized 10-20 keV emission. Since the thin accretion disk is not expected to precess and dominates the soft X-ray emission, low-energy $(<10 \mathrm{keV})$ observations are poorly poised to address this question, while Pol$S T A R$ 's broadband sensitivity can establish the physical basis of the QPO phenomenon as predicted by the calculations of Ingram et al. (2015).

GRS 1915+105 displays very strong QPOs with periods ranging from $\sim 10-0.1 \mathrm{~s}$ (e.g. Zhang et al., 2015b, and references therein). The brightness of the source, and the amplitude of the QPOs peak when the QPO period is $t_{\mathrm{qpo}} \sim 1 \mathrm{~s}$. However, accurate time resolved polarization measurements require fairly large time bins, and therefore this favors longer QPO periods. We choose an example of $t_{\mathrm{qpo}}=2 \mathrm{~s}$ as these considerations balance well for this period. PolSTAR measures a count 


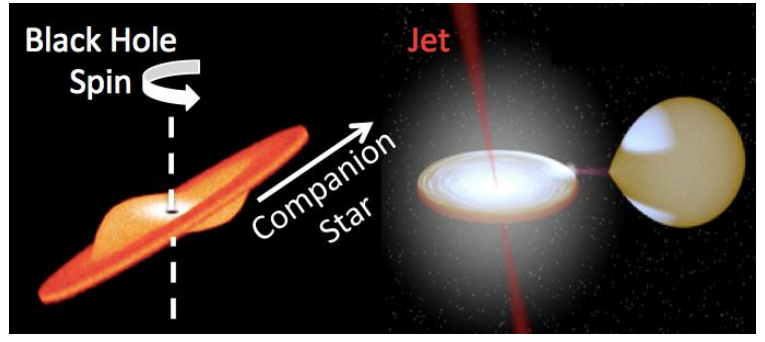

Figure 15: PolSTAR can use polarization to measure the orientation of the inner accretion disk of stellar mass black holes with an accuracy of a few degrees. Left: Pol$S T A R$ can measure the amount of misalignment between the inner disk and the binary plane owing to the general relativistic Bardeen-Peterson effect (warped accretion disk image from Lodato and Price, 2010). Right: PolSTAR is also sensitive to misalignments between the disk spin axis and the jet axis. (image from Rob Hynes, www.phys.lsu.edu/ rih/).

rate of $\sim 70 \mathrm{cs}^{-1}$ from GRS $1915+105$. We assume the modulations in flux, polarization degree and polarization angle calculated by Ingram et al. (2015) for an inclination angle of $i=70^{\circ}$, which is appropriate for GRS 1915+105.

We simulate the QPOs taking into account that they are quasi-periodic as opposed to periodic and are observed coincident with broadband noise, which is intrinsic to the source rather than instrumental. We simulate the phase of the QPO to drift on a random walk away from that of a strictly periodic sine wave (Figure 16, top panel), as is observed for QPOs in GRS 1915+105 (Morgan et al., 1997). We also simulate noise, which has a broad Lorentzian power spectrum but exhibits the statistical correlations observed in the light curves of accreting objects (the so-called linear rms-flux relation: Uttley and McHardy, 2001). To do this we use the exponentiation method of Uttley et al. (2005). We multiply the QPO and broad band noise light curves together (again to mimic statistical properties observed in the data: Ingram and van der Klis, 2013) to obtain the expected number of photon counts per time bin. Finally, we simulate PolSTAR detecting an integer number of photons per time bin by choosing a Poisson random variable from the calculated expectation value for every time bin. For each photon in each time bin, we then simulate where that photon lands on the PolSTAR detector based on
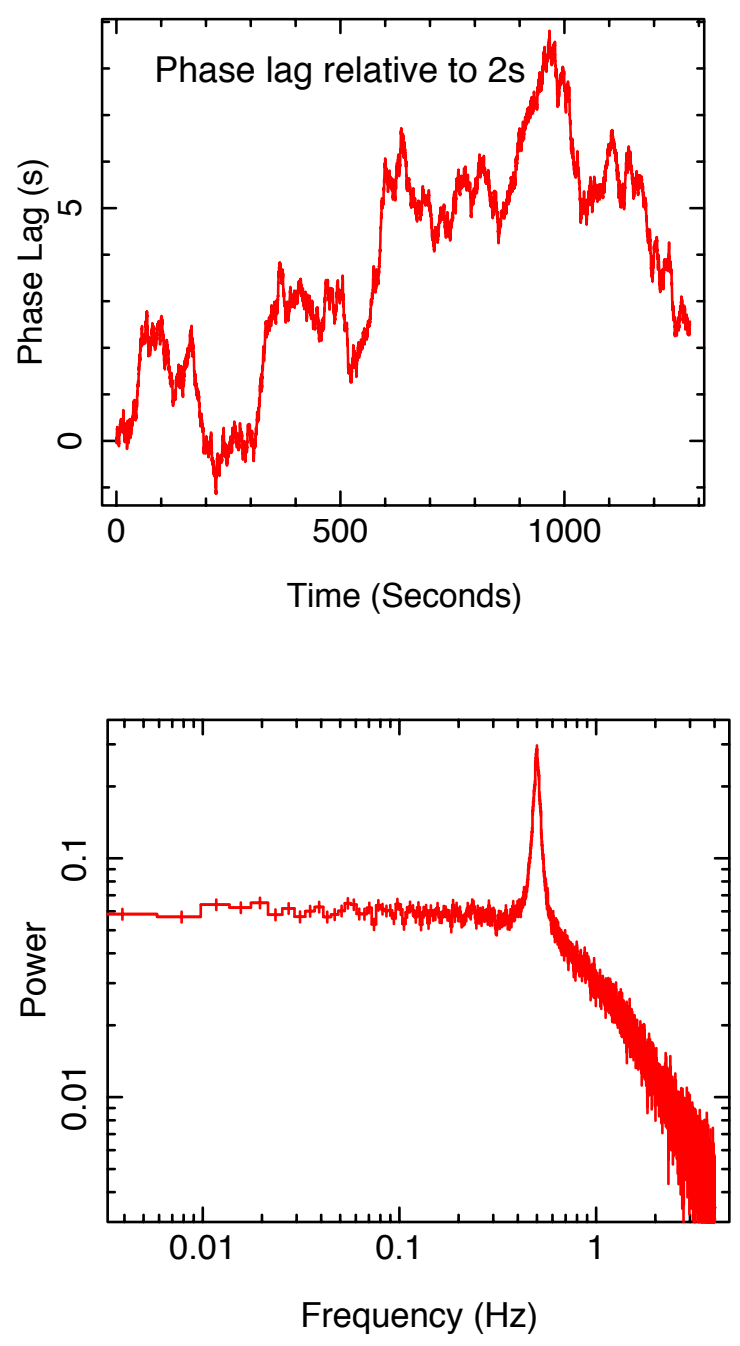

Figure 16: Top: Phase of a simulated QPO relative to a perfectly periodic function. It is this phase drift that makes QPOs quasi-periodic. Bottom: Power spectrum of the simulated light curve. The QPO is seen as a peak at $0.5 \mathrm{~Hz}$. When fit with a Lorentzian function, the QPO is measured to have a quality factor of $\mathrm{Q}=12.5$, consistent with what is observed for GRS $1915+105$.

the true polarization vector at that time and the modulation factor of PolSTAR, $\mu=0.5$.

The lower panel of Fig. 16 shows the power spectrum of the $205 \mathrm{ks}$ simulated light curve. We see a QPO and broadband noise, consistent with real observations. Measurement of Stokes parameters in $0.125 \mathrm{~s}$ time bins are too noisy to discover a QPO in the polarization signature by simply taking a power spectrum of a Stokes parameters time series. We use the phase-folding method of Tomsick and Kaaret (2001): we filter timescales much longer and shorter than the QPO period out of the flux time series and identify peaks in the 


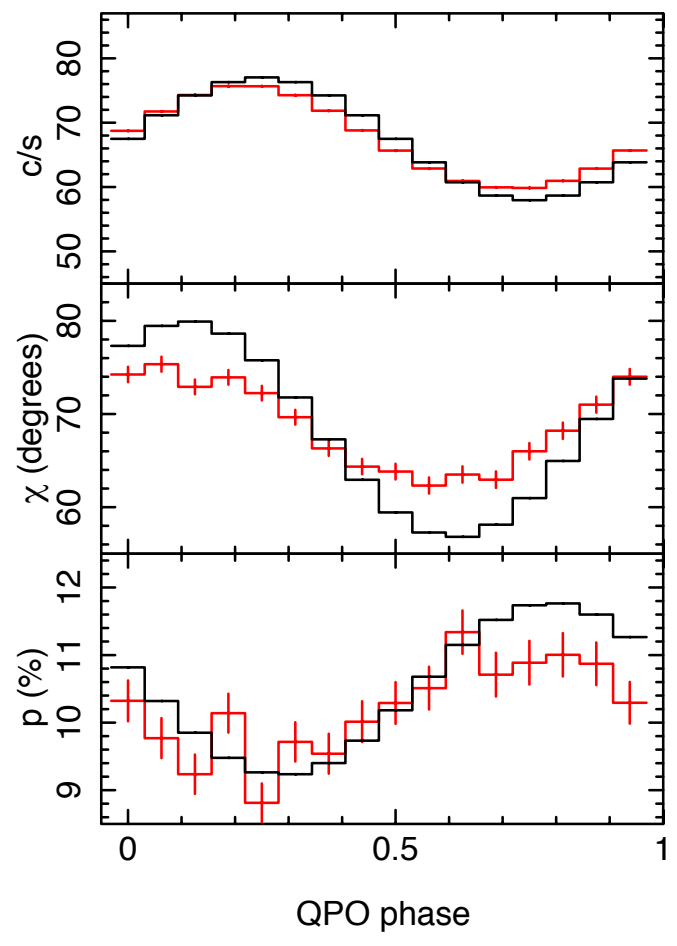

Figure 17: Count rate, polarization angle and polarization degree plotted against QPO phase. Red points show the values based on reconstructing the simulated PolSTAR data, and the black lines show the input values. Constant polarization degree and angle can be rejected with a high statistical confidence.

filtered time series as QPO peaks. We then assign a QPO phase value to every time bin based on the time since the last peak relative to the time until the next peak. We then stack into 16 QPO phase bins. Figure 17 shows the mean count rate (top), polarization angle (middle) and polarization degree (bottom) in each phase bin. Red points are recovered from the data and black lines are the input models. We see that the modulations in both polarization degree and angle are recovered and constant polarization properties can be ruled out with a high statistical significance. PolSTAR can thus detect the precession of the inner accretion flow. The PolSTAR detection would constrain accretion disk and corona models and would confirm a strong-field prediction of general relativity (e.g. Abramowicz, 2005).

\section{Numerical Modeling of the Data}

The studies of black hole accretion disks, corona, and jets would benefit and be in di-

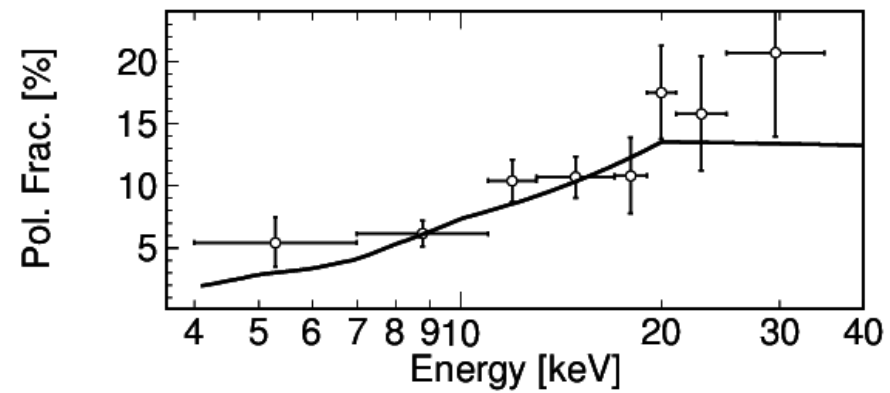

Figure 18: PolSTAR is sensitive at high energies (5-40 $\mathrm{keV}$ ), where the reflected coronal emission is highly polarized. This plot shows that advantage for a simulated 544 ksec PolSTAR observation of MCG-5-23-16 (model from: Dovčiak et al., 2011, assuming a lamppost corona).

alog with the rapidly progressing field of numerical simulations of accreting black hole systems. General relativistic magnetohydrodynamic simulations can provide a self-consistent physical model for accretion flows and jets (e.g., De Villiers and Hawley, 2003; Gammie et al., 2003) and can be used to derive testable predictions. Simulations predict that ordered magnetic fields with large fluxes (area time magnetic field strength) lead to more powerful jets (Tchekhovskoy et al., 2011), and that black holes with misaligned spin and accretion disk axes may lead to twisted jets (McKinney et al., 2013). Both of these predictions can be tested with PolSTAR data.

Recent improvements in radiation physics (Sadowski and Narayan, 2015b,a; McKinney et al., 2014; Jiang et al., 2014) and thermodynamics (Schnittman et al., 2013; Zhu et al., 2015) at high luminosities, and radiative cooling (Ryan et al., 2015) and plasma physics (Chandra et al., 2015) at low luminosities can soon be combined with radiative transfer codes (e.g., Schnittman and Krolik, 2013; Shcherbakov et al., 2012). Such general relativistic radiation magnetohydrodynamic codes make it possible to derive energy spectra, light curves, and polarization for the vast majority of the observed accreting black holes, including the PolSTAR targets. Detailed comparisons of simulated and observational (PolSTAR and other) data test the numerical models, and can be used to derive more robust constrains on parameters such as the mass accretion rate, the mag- 
netic field strength and configuration, the black hole spin, and the alignment between the accreting material and the black hole spin axes.

\subsection{Dissect the Black Hole Accretion Flows onto Supermassive Mass Black Holes}

Accretion is key in understanding how black holes grow and influence the galaxies in which they reside. The energy released from accretion on to a supermassive black hole is 100 times greater than the gravitational binding energy of its host galaxy, and yet the mass of the black hole is 1000 times less than that of the galaxy's bulge. Most of the energy released in accretion is concentrated within a few tens of gravitational radii from the central black hole. This corresponds to microparsec scales, which are far smaller than the angular resolution power of current or future telescopes. Therefore, we must rely on other properties of the emission in order to understand the geometry and kinematics of the complex regions around supermassive black holes.

Traditional spectral analysis has revealed two clear components that help us characterize the inner accretion flow: the broad $\sim 5-8 \mathrm{keV}$ Fe K- $\alpha$ emission line and the strong Compton hump component above $10 \mathrm{keV}$. These two spectral features are produced through the irradiation of the inner accretion disc by the X-ray corona, and are broadened due to relativistic effects in the strong potential well of the central black hole. NuSTAR, with its large effective area and broad energy coverage, has been ideal for measuring these two important spectral components, which has allowed for the most precise measurements of black hole spin to date (e.g. Risaliti et al., 2013; Walton et al., 2014; Marinucci et al., 2014a; Parker et al., 2014). Furthermore, NuSTAR measurements of the reverberation time delays associated with the broad Fe K- $\alpha$ line and Compton hump indicate a small light travel distance between the continuum emitting corona and the inner accretion disc (Zoghbi et al., 2014; Kara et al., 2015).

While reverberation mapping has given us a new way to constrain the accretion flow, there is still a fundamental degeneracy between the height of the corona above the accretion disc and the mass of the black hole (Cackett et al., 2014). Pol$S T A R$ could break that degeneracy. The polarization fraction and angle associated with the broad Fe K- $\alpha$ line and Compton hump are highly dependent on the height of the corona and are massinvariant. The Compton hump is particularly vital in helping break this degeneracy because the polarization fraction increases from $<1 \%$ at $3 \mathrm{keV}$ to $15 \%$ at $>20 \mathrm{keV}$ (Figure 18, Dovčiak et al., 2011). By combining spectral, timing, and polarization, PolSTAR can constrain coronal parameters such as spatial extent, relative location, optical thickness and clumpiness of the corona (e.g. Schnittman and Krolik, 2010).

The observation plan includes three of the brightest bare Seyfert galaxies, NGC 4151, MCG 5-23-16, and MCG 6-30-15. They have been observed with $N u S T A R$ and show strong Compton hump components (Keck et al., 2015; Baloković et al., 2015; Marinucci et al., 2014b). All three sources are also highly variable making them ideal targets for PolSTAR's simultaneous spectral, reverberation and polarization capabilities. XMMNewton observations of NGC 4151 and MCG-523-16 already reveal strong Fe $\mathrm{K}-\alpha$ reverberation lags, and NuSTAR observations of MCG-5-23-16 reveal the associated lag of the Compton hump (Zoghbi et al., 2012, 2014). MCG-6-30-15 is of particular interest. While it is highly variable, the continuum emission does not appear to vary with the broad Fe K- $\alpha$ emission, and therefore reverberation is not detected (Vaughan et al., 2003; Papadakis et al., 2005; Kara et al., 2014). Miniutti et al. (2003) suggests that this behaviour is due to strong relativistic light bending effects from a corona within a few gravitational radii of the black hole. PolSTAR can test this conjecture since the scenario predicts large polarization degrees for the reflected emission. The observed data can also be compared to simulated data from general relativistic radiation magnetohydrodynamic (GRRMHD) simulations (Sa̧dowski et al., 2013; McKinney et al., 2014). 


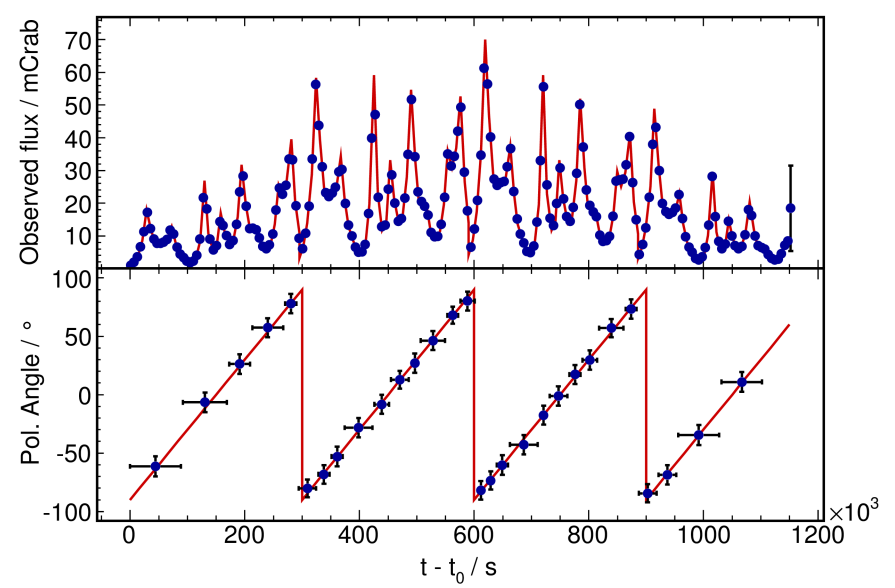

Figure 19: PolSTAR observations of blazars can reveal the helical magnetic field by detecting swings of the polarization angle. This simulation of PolSTAR observations of a Mrk 421 flaring epoch assumes a polarization fraction of $6 \%$ (similar to what is measured at optical wavelengths, e.g., Tosti et al., 1998; Blasi et al., 2013).

\subsection{Reveal the Magnetic Back-bone of Blazar Jets}

Active galactic nuclei (AGN) can launch extremely powerful and highly relativistic out-flows (jets). These jets appear to play an important role in galaxies and galaxy clusters as they can heat the interstellar and intracluster medium and thus impact the rate at which gas cools to form stars and feed the central black hole (e.g. Fabian, 2012, and references therein). Although jets have been studied intensively, their governing physics is still largely unknown (Boettcher et al., 2012). Over the last decade, the magnetic model of jet formation has emerged as the standard paradigm to explain the observed jet characteristics and relativistic velocities (Spruit, 2011, and references therein). The model invokes a helical magnetic field threading the jet. Magnetic stresses along the field lines accelerate the jet material to velocities close to the speed of light and help collimate the jets. General relativistic magnetohydrodynamic simulations seem to confirm that magnetically-dominated jets can form from the accretion process and propagate to large distances (De Villiers et al., 2005; McKinney, 2006; McKinney and Blandford, 2009). PolSTAR observations of blazars can test this model. In high synchrotron-peaked blazars (HSPs), the X- ray emission is produced by synchrotron emission and is polarized perpendicular to the projection of the magnetic field lines onto the plane of the sky. PolSTAR's observation plan includes the two bright HSPs, Mrk 421 and Mrk 501.

Optical blazar polarimetry occasionally reveals swings of the polarization angle correlated with multiwavelength flaring activity (e.g. Marscher et al., 2008; Abdo et al., 2010). Models predict that X-ray polarimetric observations show such swings more consistently than optical observations since the X-ray emitting regions are more compact (yet still optically thin) because highenergy electrons lose their energy on shorter time scales. Figure 19 illustrates simulated PolSTAR observations of a Mrk 421 flaring epoch, assuming a polarization fraction of $6 \%$ (similar to what is measured at optical wavelengths; Tosti et al., 1998; Blasi et al., 2013). It clearly shows that Pol$S T A R$ has sufficient sensitivity to detect such polarization angle swings (Figure 19), which would provide clear evidence for a helical magnetic field topology (Zhang et al., 2014, 2015a).

Even if PolSTAR does not detect ubiquitous polarization swings, it can still constrain magnetic fields inside jets (Krawczynski et al., 2013). If flares are associated with the shock-compression of magnetic fields, a correlation of X-ray flux and polarization fraction is predicted. Relatedly, correlations between the X-ray spectral index and polarization fraction constrain the magnetic field homogeneity in the emitting region. Finally, multiwavelength studies, comparing simultaneous optical and X-ray polarization measurements, provide information on the co-spatiality of the optical and X-ray emitting regions. This is an essential ingredient for modeling blazar physics.

The PolSTAR baseline program includes two flat-spectrum radio-loud quasars (FSRQs), 3C 273 and PKS 1510-08. These observations can help solve a second long-standing question in the blazar community by distinguishing between the two main flavors of radiation models to explain the X-ray to gamma-ray emission: leptonic models (including both synchrotron self-Compton and external Compton models) vs. hadronic models. As hadronic models predict higher polariza- 
tion fractions (up to $\sim 70 \%$ in the case of a perfectly ordered magnetic field in the high-energy emission region) than leptonic models (less than $\sim 30 \%$ ), a measurement of very high X-ray polarization fractions would vindicate the hadronic model (Krawczynski, 2012; Zhang and Böttcher, 2013). This in turn would imply that blazars can accelerate ions to $>10^{19} \mathrm{eV}$, comparable to the energies of ultra-high energy cosmic rays.

\subsection{Study of Extremely Magnetized Neutron Stars}

The observation plan includes two AXPs and one SGR. Both types of sources are explained by the magnetar hypothesis as neutron stars with extremely strong $\left(10^{14}-10^{15} \mathrm{G}\right)$ magnetic fields (Duncan and Thompson, 1992). The bright flares from this class of objects are thought to be magnetically powered events in which the field stresses deform or break the stellar crust, releasing a large amount of energy which leads to a reconfiguration of the magnetosphere (Thompson and Duncan, 1995). The magnetic fields are so strong that they lead to a unique phenomenology with telltale observational signatures, both for flare emission, and also for the steady, persistent signal that Pol$S T A R$ can focus on through pointed observations.

The quiescent thermal $(<4 \mathrm{keV})$ X-ray emission from magnetars is predicted to be nearly $100 \%$ polarized since the extremely magnetized plasma near the neutron star surface is birefringent, with the lowest opacity mode carrying most of the radiation (e.g., Özel 2001; Lai and Ho 2003). The non-thermal, low-energy X-rays ( 4$10 \mathrm{keV}$ ) are thought to be produced by resonant cyclotron/Compton upscattering of thermal photons in the inner magnetosphere, a process enabled by extremely strong magnetic fields. The scattering process is expected to impart a moderate polarization amplitude $(\sim 10-30 \%)$ to the outgoing photons, provided the observer samples viewing perspectives at significant angles to the local field direction. Phase-averaged observations by PolSTAR can test this basic emission mechanism since they will provide a polarization signal averaged over a variety of instantaneous magnetic viewing angles (Figure 20).

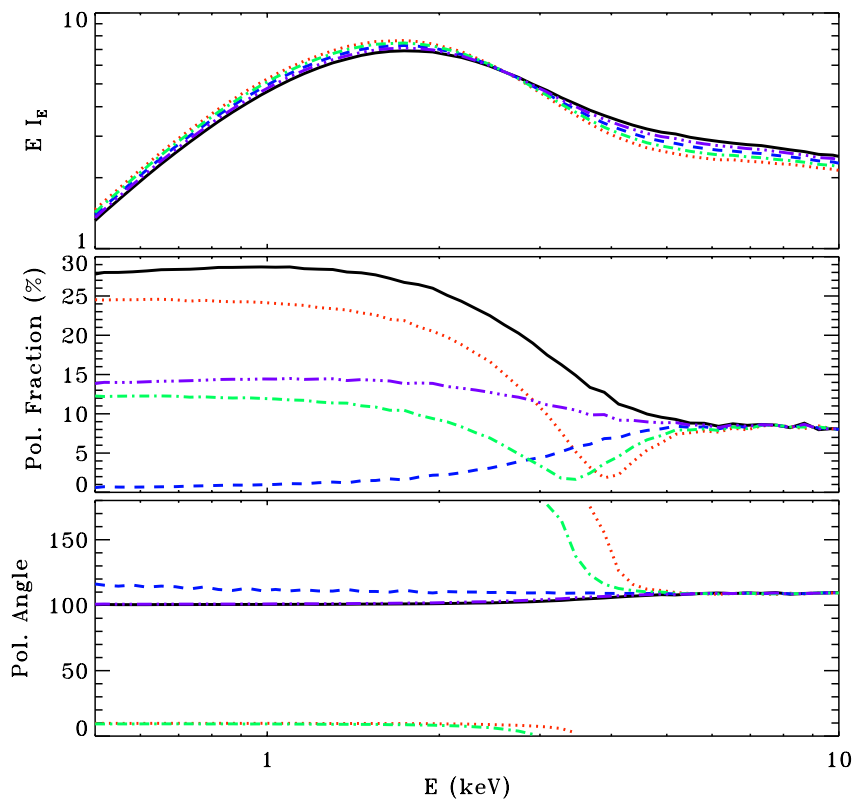

Figure 20: Intensity (top), linear polarization fraction (middle), and polarization angle (bottom) as a function of energy for phase-averaged observations. The magnetar model predicts that the soft X-ray emission is a combination of highly polarized thermal photons at energies $<4 \mathrm{keV}$, with resonant Compton upscattering providing a non-thermal tail at energies $>4 \mathrm{keV}$. The different lines shows predictions for different polarization properties of the thermal seed photons from the magnetar surface (from Fernández and Davis, 2011, CAAS, reproduced with permission).

PolSTAR's detection of the extremely polarized ( $\sim 30-80 \%)$ phase-resolved emission predicted by the magnetar model would distinguish between alternatives such as the fallback accretion model, which attributes the non-thermal emission to a combination of thermal and bulk Compton scattering in the accretion flow (Trümper et al., 2010, $2013)$, leading to lower $(<10 \%)$ polarization fractions (Figure 21). This low expectation is an estimate based on non-magnetic Comptonization scenarios in laminar geometries, for which computed polarization degrees typically in this range were obtained by Sunyaev and Titarchuk (1985) in the context of black hole accretion disks.

The phase-resolved $3-15 \mathrm{keV}$ polarization angle measurements can distinguish between a pure dipole field geometry and the twisted magnetic field at the heart of the magnetar model 


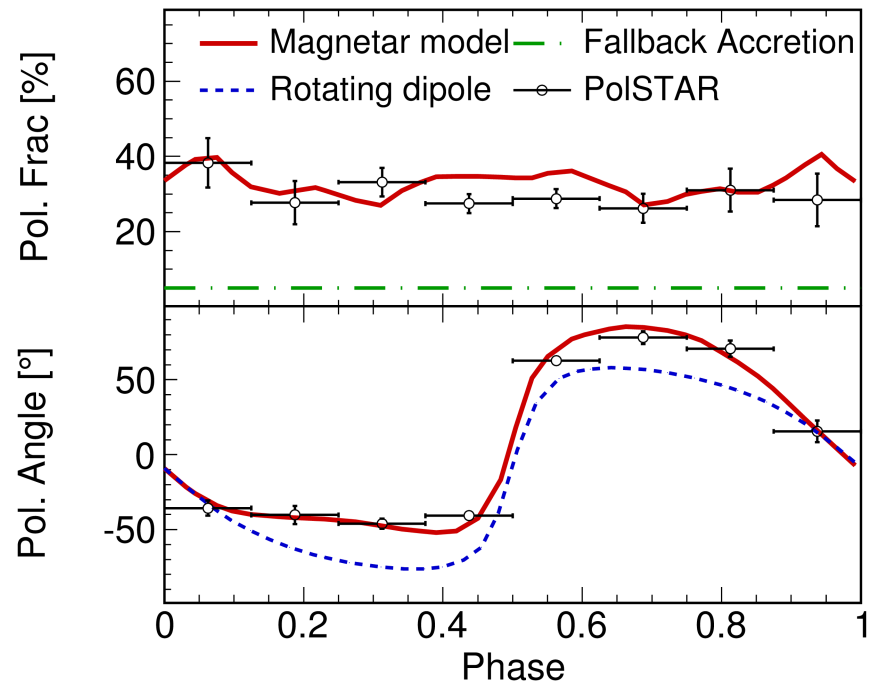

Figure 21: PolSTAR observations of the AXP 1E $2259+586$ can distinguish the magnetar model (top panel, red line) from the fallback accretion model (top panel, green line). Both panels use the $3-15 \mathrm{keV}$ data. The lower panel compares the modeled polarization direction (red line) and PolSTAR measurements (black data points) to the time-reversed model (blue dashed line). The detection of such a time reversal asymmetry implies the presence of a toroidal magnetic field component in the magnetosphere. The magnetar model predictions are from Fernández and Davis (2011). No detailed predictions for the fallback model are available, but modest $(\lesssim 10 \%)$ values are expected if polarization arises from Compton scattering as is assumed in the fallback model (Trümper et al., 2010).

(Fernández and Davis, 2011). This is because each phase corresponds to a particular viewing orientation relative to the mean magnetic field direction sampled in the emission region, for either surface/atmospheric signals or radiation generated in the inner magnetosphere. PolSTAR can contrast polarization signatures (fraction and direction) between quiescent and post-outburst states, and search for evidence of a magnetic reconfiguration initiated by the outburst activity. At even higher $(>10 \mathrm{keV})$ energies, Pol$S T A R$ can detect the flat hard X-ray tails of magnetars (Kuiper et al., 2004; Götz et al., 2006; den Hartog et al., 2008) believed to be polarized to $>50 \%$ owing to resonant inverse Compton/cyclotron scattering (Baring and Harding, 2007). In such upscattering models, the polar-

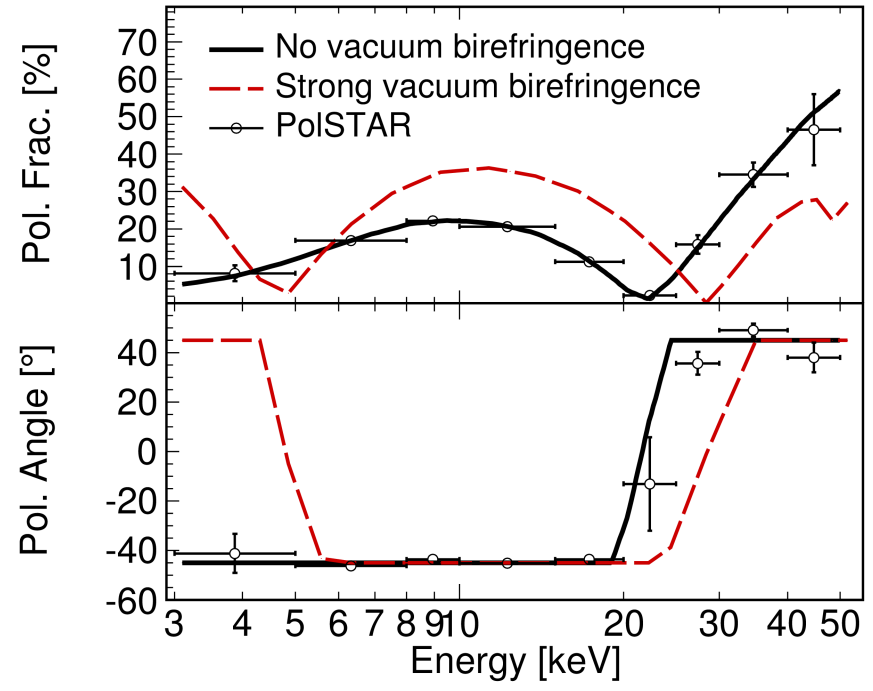

Figure 22: PolSTAR is sensitive to the distinct polarization signatures of QED vacuum birefringence. This simulated 22 ksec PolSTAR observation of Her X-1 in its highstate distinguishes between the model with (dashed red line) and without (solid black line) vacuum birefringence. The models predictions are from Kii (1987).

ization degree increases at higher photon energies due to an increased contribution from photons backscattered in an electron's rest frame.

The PolSTAR observations of magnetars and accreting pulsars can probe the dielectric properties of the strongly magnetized quantum vacuum to test predictions of the theory of Quantum Electrodynamics (QED) in extremely strong magnetic fields not accessible in terrestrial laboratories. At field strengths above the quantum critical field, $4.41 \times 10^{13} \mathrm{G}$, the vacuum itself is polarized by QED coupling to virtual $e^{+} e^{-}$ pairs, thereby rendering the magnetosphere birefringent, i.e., the refractive index differs for the two photon polarizations (e.g. see Harding and Lai, 2006; Fernández and Davis, 2011). X-ray photons of different polarizations therefore travel at slightly different speeds, and their electric field vectors can re-orient during propagation through the magnetosphere, rotating about the local magnetic field direction. This effect is more pronounced in magnetars than for pulsars of lower magnetization.

While magnetars serve as the best type of neu- 
tron stars with which to study magnetospheric propagation influences of vacuum birefringence, accreting pulsars with much lower magnetic field strengths of around $10^{12} \mathrm{G}$ present another opportunity to test fundamental QED predictions of dispersion of the magnetized vacuum. This is in reference to the so-called vacuum resonance feature, discussed in detail in the review by Harding and Lai (2006). This corresponds to the critical photon frequency $\omega$ where the vacuum modifications to the refractive index $n$ (which depend only on the magnetic field $B$ ) approximately equal the dispersion correction imposed by a plasma, which scales as $\omega_{p}^{2} / \omega^{2}$, where $\omega_{p}$ is the familiar plasma frequency. The vacuum resonance frequency therefore depends on the plasma density and the magnetic field strength. Accretion columns impacting neutron stars provide density and $B$ values for which the feature naturally appears above a few $\mathrm{keV}$. In contrast, magnetars will elicit such a feature in their atmospheres at energies below $1 \mathrm{keV}$, a band that generally leads to obscuration of its signatures.

As the photon frequency rises through the resonance from plasma to vacuum dispersion domains, the character of the photon propagation eigenstates changes profoundly, leading to distinctive swings in polarization degree and angle (Kii, 1987; Mészáros, 1992) when integrated over emission volumes. PolSTAR is well-suited to conduct a search for QED signatures in the $5-50 \mathrm{keV}$ polarization spectra of accreting pulsars at the energies of the vacuum resonance and cyclotron lines where these effects are strongest (see Figure 22 for examples). Observational demonstration of the existence of the vacuum resonance feature through X-ray polarimetry would be a huge advance for the fundamental theory of QED in strong field domains.

\subsection{Observations of Accretion Powered Neutron Stars and Pulsars}

The nature of the accretion flow onto neutron stars with lower surface magnetic fields (e.g. $B \leq$ $10^{9}$ Gauss) remains uncertain, despite decades of $\mathrm{X}$-ray timing and spectroscopy. Persistently accreting neutron stars trace well-known "Z" and "atoll" tracks in X-ray color-color diagrams over time (Hasinger and van der Klis, 1989), indicating that accretion is somehow regulated. Both strong quasi-periodic oscillations tied to the inner accretion disk and relativistic radio jets change in characteristic ways along these tracks (Migliari and Fender, 2006). It is likely the case that the $\mathrm{Z} /$ atoll tracks, QPOs, and jet production are all affected or even driven by the interaction of the neutron star magnetic field with accreting matter, but strong evidence of this has remained elusive. By sensitively searching for changes in polarization fraction and angle along color-color tracks, PolSTAR brings an entirely new diagnostic tool to bear on neutron star accretion. Observations of bright sources such as Scorpius X-1 and Cygnus $\mathrm{X}-2$, among others, can achieve this science within reasonable observing times.

PolSTAR observations of accretion-powered pulsars can demonstrate the cyclotron nature of the hard X-ray absorption lines seen in neutron stars based on energy-dependent polarization measurements across the lines. PolSTAR's energy resolution should be sufficiently good as the cyclotron lines exhibit typical widths of 5$10 \mathrm{keV}$. The results refine neutron star magnetic field strength measurements and probe the geometry of the accretion shock (and the particle acceleration region) by distinguishing between pencil beam and fan beam approximations to the accretion column geometry (Mészáros, 1992).

\subsection{Observations of the Crab and Vela Pulsars and Pulsar Wind Nebulas}

The rotation powered Crab pulsar and nebula remains a prime target of high-energy astrophysical research. The system is a paragon of a high-energy astrophysical source, e.g. AGN and GRB models are based on models developed for explaining Crab observations. The recent discovery of flares with the AGILE and Fermi gamma-ray telescopes (Tavani et al., 2011; Abdo et al., 2011) may require a re-evaluation of the basic assumptions about the physical processes accelerating high-energy emitting particles in these sources (Clausen-Brown and Lyutikov, 2012; Cerutti et al., 2012; Lyubarsky, 2012). As 
one of the brightest sources in the X-ray sky (flux of $3 \times 10^{-8} \mathrm{erg} \mathrm{cm}^{-2} \mathrm{sec}^{-1}$ in the $1-10 \mathrm{keV}$ range, Kellogg, 1971), it is the only one for which X-ray polarization has been measured with a high degree of confidence (Weisskopf et al., 1978).

PolSTAR would observe the Crab and Vela pulsars and pulsar wind nebulae. Although PolSTAR does not have imaging capabilities, phase-resolved analyses can be used to constrain the polarization properties of the magnetospheric emission. The phase- and energy-resolved polarization fraction and angle can discern synchrotron and curvature emission (because of their contrasting position angle sweeps with pulse phase) and provide excellent diagnostics on the locale of the magnetospheric emission (see Dyks et al., 2004, and references therein). PolSTAR is not able to image the polarization properties of the nebular emission. A next-generation imaging X-ray polarimeter with one to two orders of magnitude higher throughput than PolSTAR, would be able to access the polarization properties of the innermost particle acceleration regions. Recent measurements of $60 \%$ optical polarization of the inner knot - (Moran et al., 2013) significantly exceeded theoretical expectations (Rudy et al., 2015). This suggests filamentary field structure as opposed to a more chaotic morphology. Discerning differences between optical and X-ray polarization in the nebula would yield fascinating insights into the scaledependence of the field geometry.

\section{Summary}

The PolSTAR X-ray polarimeter offers the capability to measure the linear polarization of $\mathrm{X}$ rays over the broad $2.5-70 \mathrm{keV}$ energy range. The approach combines a $\mathrm{Si} / \mathrm{W}$ multilayer coated Xray mirror assembly with a scattering element and CZT solid state detectors, all covering the 2.5-70 $\mathrm{keV}$ energy range. The combination leads to a high $\mathrm{O}(100 \%)$ efficiency at energies exceeding 10 $\mathrm{keV}$. The modest scattering efficiency at $<10 \mathrm{keV}$ energies is offset by the large and approximately constant modulation factor of $\mu \approx 0.5$. For the brightest objects of the baseline observation program (accreting stellar mass black holes, neutron

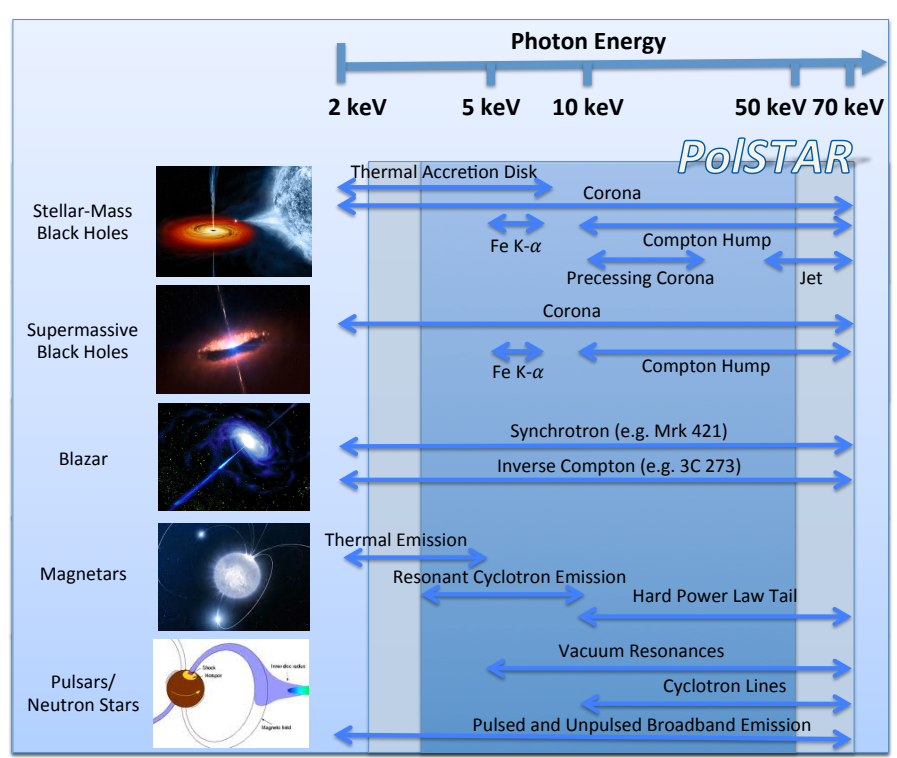

Figure 23: PolSTAR covers the most important emission components of accreting black holes, blazars, and accretion, rotation, and magnetically powered neutron stars. The measurement of the polarization properties of several emission components allows for powerful tests of the leading models.

stars, blazars), PolSTAR delivers data sets with very high signal to noise ratios allowing dynamic studies of such phenomena as QPOs and blazar flares. Even for the dimmest of the objects of the baseline observation program (AGNs and magnetars), the polarization measurements can answer important astrophysical questions.

A scattering polarimeter like PolSTAR offers a broad energy range covering the most important emission components of black holes, jets, magnetars and neutron stars (Figure 23). Simultaneous observations of multiple emission components are not only crucial for disentangling the polarization properties of the individual components, but also for measuring the relative polarization angles and thus the relative orientation of different emission regions. The measurements of the polarization of key accreting black hole components can be used to tighten existing constraints on the black hole parameters (including the black hole spin) and to study the corona whose geometry is poorly under- 
stood and plays an important role in X-ray spectroscopy and timing black hole studies. Furthermore, the hard X-ray coverage has the potential to reveal the origin of low-frequency QPOs and possibly to detect Lense-Thirring precession in the extreme spacetime of rapidly spinning black holes. The observations of blazar jets have the potential to validate the paradigm that helical magnetic fields accelerate and collimate AGN jets. The observations of magnetars enable the confirmation of the magnetar paradigm and the detection of polarization from resonant cyclotron scattering. Furthermore, a mission like PolSTAR can scrutinize the emission for imprints of the birefringent $\mathrm{QED}$ vacuum. The science described here can best be addressed with a broad energy bandpass polarimeter like PolSTAR. As mentioned in the introduction, we are considering proposing a somewhat larger version of PolSTAR to the next MIDEX announcement of opportunity.

At the time of writing this article we are preparing a first one-day long science flight of the balloon-borne $X$-Calibur experiment planned for Fall 2016. As mentioned above, $X$-Calibur is (like PolSTAR) a scattering polarimeter. The one-day flight should allow us to observe the Crab, the stellar mass black holes GRS 1915+105 and Cyg $\mathrm{X}-1$, and the accreting neutron star Sco X-1. Depending on the flux state of the sources during the observations, $X$-Calibur will be able to measure the polarization of the $30-60 \mathrm{keV}$ X-ray emission down to $5-10 \%$ polarization fractions. The most significant results of the one-day flight will be the polarization properties of the power law (presumably corona) emission from GRS $1915+105$ and Cyg X-1, and the energy resolved polarization properties of the Crab emission. The one-day balloon flight will be followed up by a $\sim 28$-day long LDB (long duration balloon) flight from McMurdo (Ross Island) in December 2018-January 2019. The longer flight will be used to measure the X-ray polarization of a sample of accreting $\mathrm{X}$-ray pulsars, flaring X-ray binaries, and the extragalactic radio galaxy Cen $\mathrm{A}$.

X-ray polarimetry missions can build on the successes of the past and current X-ray missions and are complementary to future X-ray missions.
For example, traditional X-ray spectroscopy observations have revealed the broad Fe K- $\alpha$ line at $\sim 5-8 \mathrm{keV}$ and the Compton hump above $10 \mathrm{keV}$ in the energy spectra of stellar mass and supermassive black holes (e.g. Reynolds, 2014, and references therein). These features can be well described through a relativistic reflection model in which an irradiated inner accretion disc produces fluorescence and Compton scattering that appears to the observer to be blurred due to relativistic effects close to the central black hole (Fabian et al., 1989; Ross and Fabian, 1993). The AGN Fe K$\alpha$ observations constrain black hole spins to $\sim 1 \%$ statistical uncertainty (e.g. Risaliti et al., 2013; Marinucci et al., 2014a). Recently, AGN X-ray reverberation mapping has indeed confirmed the basic premises of this model (Kara et al., 2013; Zoghbi et al., 2014; Uttley et al., 2014). X-ray polarimetry would allow us to further refine our knowledge about the geometry of the inner flow, and thus improve on the systematic uncertainties associated with the spin measurements (Dovčiak et al., 2004, 2008; Li et al., 2009; Schnittman and Krolik, 2009, 2010; Dovčiak et al., 2011). These measurements are of particular interest in light of ESA's planned Athena mission (planned to be launched in 2028) (e.g. Wilms et al., 2014). Athena, with unprecedented effective area $\left(2 \mathrm{~m}^{2}\right.$ at $1 \mathrm{keV}$ and $0.25 \mathrm{~m}^{2}$ at $6 \mathrm{keV}$ ), has the ability to put strong constraints on the broad iron line in a large sample of supermassive black holes out to a redshifts of 2 or 3. Measuring the distribution of spin in high-redshift quasars constrains the growth of supermassive black holes in the universe (e.g. Berti and Volonteri 2008). The X-ray polarimetric observations of AGNs in our local Universe would directly benefit those studies.

\section{Acknowledgements}

HK thanks the McDonnell Center for the Space Sciences at Washington University for sponsoring the design and fabrication of the X-Calibur prototype polarimeter, and NASA grant \#NNX14AD19G for the support of the X-Calibur balloon project. MGB acknowledges NASA grants NNX13AQ82G and NNX13AP08G 
for support. CD acknowledges STFC support from grant ST/L00075X/1. JCM acknowledges NASA/NSF/TCAN grant NNX14AB46G, NSF/XSEDE/TACC (TGPHY120005), and NASA/Pleiades (SMD-14-5451). AI acknowledges support from the Netherlands Organization for Scientific Research (NWO) Veni Fellowship. RF acknowledges support from the University of California Office of the President, and from NSF grant AST-1206097.

\section{References}

Abdo, A. A., Ackermann, M., Ajello, M., et al., Feb. 2011. Gamma-Ray Flares from the Crab Nebula. Science 331, 739 -.

Abdo, A. A., Ackermann, M., Ajello, M., et al., Feb. 2010. A change in the optical polarization associated with a $\gamma$-ray flare in the blazar 3C279. Nature463, 919-923.

Abramowicz, M. A., Nov. 2005. QPO as the Rosetta Stone for understanding black hole accretion. Astronomische Nachrichten 326, 782-786.

Agostinelli, S., Allison, J., Amako, K., et al., Jul. 2003. GEANT4 - a simulation toolkit. Nuclear Instruments and Methods in Physics Research A 506, 250-303.

Angel, J. R. P., Oct. 1969. Polarization of Thermal X-Ray Sources. ApJ158, 219.

Bachetti, M., Harrison, F. A., Cook, R., et al., Feb. 2015. No Time for Dead Time: Timing Analysis of Bright Black Hole Binaries with NuSTAR. ApJ800, 109.

Bachetti, M., Harrison, F. A., Walton, D. J., et al., Oct. 2014. An ultraluminous X-ray source powered by an accreting neutron star. Nature514, 202-204.

Baloković, M., Matt, G., Harrison, F. A., et al., Feb. 2015. Coronal Properties of the Seyfert 1.9 Galaxy MCG-0523-016 Determined from Hard X-Ray Spectroscopy with NuSTAR. ApJ800, 62.

Baring, M. G., Harding, A. K., Apr. 2007. Resonant Compton upscattering in anomalous X-ray pulsars. Astrophysics and Space Science 308, 109-118.

Beilicke, M., Kislat, F., Zajczyk, A., et al., 2014a. Design and Performance of the X-ray Polarimeter X-Calibur. Journal of Astronomical Instrumentation 3, 40008.

Beilicke, M., Kislat, F., Zajczyk, A., et al., 2014b. Design and Performance of the X-ray Polarimeter X-Calibur. Journal of Astronomical Instrumentation 3, 40008.

Bellazzini, R., Costa, E., Matt, G., Tagliaferri, G., Jul. 2010. X-ray Polarimetry. Cambridge University Press.

Berti, E., Volonteri, M., Sep. 2008. Cosmological Black Hole Spin Evolution by Mergers and Accretion. ApJ684, 822-828.

Blasi, M. G., Lico, R., Giroletti, M., et al., Nov. 2013. The TeV blazar Markarian 421 at the highest spatial resolution. A\&A559, A75.
Boettcher, M., Harris, D. E., Krawczynski, H., Jan. 2012. Relativistic Jets from Active Galactic Nuclei. Wiley$\mathrm{VCH}$.

Cackett, E. M., Zoghbi, A., Reynolds, C., et al., Mar. 2014. Modelling the broad $\mathrm{Fe} \mathrm{K} \alpha$ reverberation in the AGN NGC 4151. MNRAS438, 2980-2994.

Cerutti, B., Uzdensky, D. A., Begelman, M. C., Feb. 2012. Extreme Particle Acceleration in Magnetic Reconnection Layers: Application to the Gamma-Ray Flares in the Crab Nebula. ApJ746, 148.

Chandra, M., Gammie, C. F., Foucart, F., Quataert, E., Aug. 2015. An Extended Magnetohydrodynamics Model for Relativistic Weakly Collisional Plasmas. ArXiv e-prints.

Chandrasekhar, S., 1960. Radiative transfer.

Christensen, F. E., Jakobsen, A. C., Brejnholt, N. F., et al., Sep. 2011. Coatings for the NuSTAR mission. In: Society of Photo-Optical Instrumentation Engineers (SPIE) Conference Series. Vol. 8147 of Society of PhotoOptical Instrumentation Engineers (SPIE) Conference Series. p. 0.

Clausen-Brown, E., Lyutikov, M., Oct. 2012. Crab nebula gamma-ray flares as relativistic reconnection minijets. MNRAS426, 1374-1384.

Costa, E., Bellazzini, R., Soffitta, P., et al., Mar. 2006. Opening a New Window to Fundamental Physics and Astrophysics: X-ray Polarimetry. ArXiv Astrophysics e-prints.

Craig, W. W., An, H., Blaedel, K. L., et al., Sep. 2011. Fabrication of the NuSTAR flight optics. In: Society of Photo-Optical Instrumentation Engineers (SPIE) Conference Series. Vol. 8147 of Society of Photo-Optical Instrumentation Engineers (SPIE) Conference Series. p. 0.

De Villiers, J.-P., Hawley, J. F., May 2003. A Numerical Method for General Relativistic Magnetohydrodynamics. ApJ589, 458-480.

De Villiers, J.-P., Hawley, J. F., Krolik, J. H., Hirose, S., Feb. 2005. Magnetically Driven Accretion in the Kerr Metric. III. Unbound Outflows. ApJ620, 878-888.

Dean, A. J., Clark, D. J., Stephen, J. B., et al., Aug. 2008. Polarized Gamma-Ray Emission from the Crab. Science 321, 1183-.

den Hartog, P. R., Kuiper, L., Hermsen, W., et al., Oct. 2008. Detailed high-energy characteristics of AXP $4 \mathrm{U}$ 0142+61. Multi-year observations with INTEGRAL, RXTE, XMM-Newton, and ASCA. A\&A489, 245-261.

Dovčiak, M., Karas, V., Matt, G., Dec. 2004. Polarization signatures of strong gravity in active galactic nuclei accretion discs. MNRAS355, 1005-1009.

Dovčiak, M., Muleri, F., Goosmann, R. W., Karas, V., Matt, G., Nov. 2008. Thermal disc emission from a rotating black hole: X-ray polarization signatures. MNRAS391, 32-38.

Dovčiak, M., Muleri, F., Goosmann, R. W., Karas, V., Matt, G., Apr. 2011. Light-bending Scenario for Accreting Black Holes in X-ray Polarimetry. ApJ731, 75. 
Duncan, R. C., Thompson, C., Jun. 1992. Formation of very strongly magnetized neutron stars - Implications for gamma-ray bursts. ApJL392, L9-L13.

Dyks, J., Harding, A. K., Rudak, B., May 2004. Relativistic Effects and Polarization in Three High-Energy Pulsar Models. ApJ606, 1125-1142.

Enoto, T., Black, J. K., Kitaguchi, T., et al., Jul. 2014. Performance verification of the Gravity and Extreme Magnetism Small explorer (GEMS) x-ray polarimeter. In: Society of Photo-Optical Instrumentation Engineers (SPIE) Conference Series. Vol. 9144 of Society of PhotoOptical Instrumentation Engineers (SPIE) Conference Series. p. 4.

Fabian, A. C., Sep. 2012. Observational Evidence of Active Galactic Nuclei Feedback. ARA\&A50, 455-489.

Fabian, A. C., Rees, M. J., Stella, L., White, N. E., May 1989. X-ray fluorescence from the inner disc in Cygnus X-1. MNRAS238, 729-736.

Fernández, R., Davis, S. W., Apr. 2011. The X-ray Polarization Signature of Quiescent Magnetars: Effect of Magnetospheric Scattering and Vacuum Polarization. ApJ730, 131.

Forot, M., Laurent, P., Grenier, I. A., Gouiffès, C., Lebrun, F., Nov. 2008. Polarization of the Crab Pulsar and Nebula as Observed by the INTEGRAL/IBIS Telescope. ApJL688, L29-L32.

Fragile, P. C., Blaes, O. M., Anninos, P., Salmonson, J. D., Oct. 2007. Global General Relativistic Magnetohydrodynamic Simulation of a Tilted Black Hole Accretion Disk. ApJ668, 417-429.

Fukazawa, Y., Tajima, H., Watanabe, S., et al., Jul. 2014. Soft gamma-ray detector (SGD) onboard the ASTRO-H mission. In: Society of Photo-Optical Instrumentation Engineers (SPIE) Conference Series. Vol. 9144 of Society of Photo-Optical Instrumentation Engineers (SPIE) Conference Series. p. 2.

Fürst, F., Grefenstette, B. W., Staubert, R., et al., Dec. 2013. The Smooth Cyclotron Line in Her X-1 as Seen with Nuclear Spectroscopic Telescope Array. ApJ779, 69 .

Fürst, F., Pottschmidt, K., Miyasaka, H., et al., Jun. 2015. Distorted Cyclotron Line Profile in Cep X-4 as Observed by NuSTAR. ApJL806, L24.

Fürst, F., Pottschmidt, K., Wilms, J., et al., Apr. 2014a. NuSTAR Discovery of a Cyclotron Line in KS 1947+300. ApJL784, L40.

Fürst, F., Pottschmidt, K., Wilms, J., et al., Jan. 2014b. NuSTAR Discovery of a Luminosity Dependent Cyclotron Line Energy in Vela X-1. ApJ780, 133.

Gammie, C. F., McKinney, J. C., Tóth, G., May 2003. HARM: A Numerical Scheme for General Relativistic Magnetohydrodynamics. ApJ589, 444-457.

Ghosh, P., Angelini, L., Baring, M., et al., Jan. 2013. White Paper on GEMS Study of Polarized X-rays from Neutron Stars. ArXiv e-prints.

Gilfanov, M., Merloni, A., Sep. 2014. Observational Ap- pearance of Black Holes in X-Ray Binaries and AGN. Space Science Review183, 121-148.

Götz, D., Mereghetti, S., Tiengo, A., Esposito, P., Apr. 2006. Magnetars as persistent hard X-ray sources: INTEGRAL discovery of a hard tail in SGR 1900+14. A\&A449, L31-L34.

Gou, L., McClintock, J. E., Reid, M. J., et al., Dec. 2011. The Extreme Spin of the Black Hole in Cygnus X-1. ApJ742, 85.

Gou, L., McClintock, J. E., Remillard, R. A., et al., Jul. 2014. Confirmation via the Continuum-fitting Method that the Spin of the Black Hole in Cygnus X-1 Is Extreme. ApJ790, 29.

Guo, Q., Beilicke, M., Garson, A., et al., Jan. 2013. Optimization of the design of the hard X-ray polarimeter X-Calibur. Astroparticle Physics 41, 63-72.

Hailey, C. J., An, H., Blaedel, K. L., et al., Jul. 2010a. The Nuclear Spectroscopic Telescope Array (NuSTAR): optics overview and current status. In: Society of PhotoOptical Instrumentation Engineers (SPIE) Conference Series. Vol. 7732 of Society of Photo-Optical Instrumentation Engineers (SPIE) Conference Series. p. 0.

Hailey, C. J., An, H., Blaedel, K. L., et al., Jul. 2010b. The Nuclear Spectroscopic Telescope Array (NuSTAR): optics overview and current status. In: Society of PhotoOptical Instrumentation Engineers (SPIE) Conference Series. Vol. 7732 of Society of Photo-Optical Instrumentation Engineers (SPIE) Conference Series. p. 0.

Harding, A. K., Lai, D., Sep. 2006. Physics of strongly magnetized neutron stars. Reports on Progress in Physics 69, 2631-2708.

Harrison, F. A., Craig, W. W., Christensen, F. E., et al., Jun. 2013. The Nuclear Spectroscopic Telescope Array (NuSTAR) High-energy X-Ray Mission. ApJ770, 103.

Hasinger, G., van der Klis, M., Nov. 1989. Two patterns of correlated X-ray timing and spectral behaviour in low-mass X-ray binaries. A\&A225, 79-96.

Heil, L. M., Uttley, P., Klein-Wolt, M., Apr. 2015. Inclination-dependent spectral and timing properties in transient black hole X-ray binaries. MNRAS448, 33483353.

Hill, J. E., Black, J. K., Emmett, T. J., et al., Jul. 2014. Design improvements and x-ray performance of a time projection chamber polarimeter for persistent astronomical sources. In: Society of Photo-Optical Instrumentation Engineers (SPIE) Conference Series. Vol. 9144 of Society of Photo-Optical Instrumentation Engineers (SPIE) Conference Series. p. 1.

Hughes, J. P., Long, K. S., Novick, R., May 1984. A search for X-ray polarization in cosmic X-ray sources. ApJ280, $255-258$.

Ingram, A., Done, C., Fragile, P. C., Jul. 2009. Lowfrequency quasi-periodic oscillations spectra and LenseThirring precession. MNRAS397, L101-L105.

Ingram, A., Maccarone, T., Poutanen, J., Krawczynski, H., Apr. 2015. Polarization modulation from Lense- 
Thirring precession in X-ray binaries. ArXiv e-prints.

Ingram, A., van der Klis, M., Sep. 2013. An exact analytic treatment of propagating mass accretion rate fluctuations in X-ray binaries. MNRAS434, 1476-1485.

Jahoda, K., Kouveliotou, C., Kallman, T. R., Praxys Team, Jan. 2015. Polarization from Relativistic Astrophysical X-raY Sourses: The PRAXYS Small Explorer Observatory. In: American Astronomical Society Meeting Abstracts. Vol. 225 of American Astronomical Society Meeting Abstracts. p. \#338.40.

Jahoda, K., Swank, J. H., Giles, A. B., et al., Oct. 1996. In-orbit performance and calibration of the Rossi X-ray Timing Explorer (RXTE) Proportional Counter Array (PCA). In: Siegmund, O. H., Gummin, M. A. (Eds.), EUV, X-Ray, and Gamma-Ray Instrumentation for Astronomy VII. Vol. 2808 of Society of Photo-Optical Instrumentation Engineers (SPIE) Conference Series. pp. 59-70.

Jahoda, K. M., Black, J. K., Hill, J. E., et al., Jul. 2014. Xray polarization capabilities of a small explorer mission. In: Society of Photo-Optical Instrumentation Engineers (SPIE) Conference Series. Vol. 9144 of Society of PhotoOptical Instrumentation Engineers (SPIE) Conference Series. p. 0.

Jiang, Y.-F., Stone, J. M., Davis, S. W., Dec. 2014. A Global Three-dimensional Radiation Magnetohydrodynamic Simulation of Super-Eddington Accretion Disks. ApJ796, 106.

Kalemci, E., Boggs, S. E., Kouveliotou, C., Finger, M., Baring, M. G., Mar. 2007. Search for Polarization from the Prompt Gamma-Ray Emission of GRB 041219a with SPI on INTEGRAL. ApJS169, 75-82.

Kara, E., Fabian, A. C., Cackett, E. M., et al., Sep. 2013. Discovery of high-frequency iron K lags in Ark 564 and Mrk 335. MNRAS434, 1129-1137.

Kara, E., Fabian, A. C., Marinucci, A., et al., Nov. 2014. The changing X-ray time lag in MCG-6-30-15. MNRAS445, 56-65.

Kara, E., Zoghbi, A., Marinucci, A., et al., Jan. 2015. Iron $\mathrm{K}$ and Compton hump reverberation in SWIFT J2127.4+5654 and NGC 1365 revealed by NuSTAR and XMM-Newton. MNRAS446, 737-749.

Katz, J. I., Jun. 1976. Nonrelativistic Compton scattering and models of quasars. ApJ206, 910-916.

Keck, M. L., Brenneman, L. W., Ballantyne, D. R., et al., Apr. 2015. NuSTAR and Suzaku X-ray Spectroscopy of NGC 4151: Evidence for Reflection from the Inner Accretion Disk. ArXiv e-prints.

Kellogg, E. M., 1971. X-Ray Observations of the Crab Nebula. In: Davies, R. D., Graham-Smith, F. (Eds.), The Crab Nebula. Vol. 46 of IAU Symposium. p. 42.

Kestenbaum, H. L., Cohen, G. G., Long, K. S., et al., Dec. 1976. The graphite crystal X-ray spectrometer on OSO-8. ApJ210, 805-809.

Kii, T., 1987. X-ray polarizations from accreting strongly magnetized neutron stars - Case studies for the X-ray pulsars 4U 1626-67 and Hercules X-1. PASJ39, 781-800. Kislat, F., Clark, B., Beilicke, M., Krawczynski, H., Aug. 2015. Analyzing the data from X-ray polarimeters with Stokes parameters. Astroparticle Physics 68, 45-51.

Kitaguchi, T., Tamagawa, T., Hayato, A., et al., Jul. 2014. Monte-Carlo estimation of the inflight performance of the GEMS satellite x-ray polarimeter. In: Society of Photo-Optical Instrumentation Engineers (SPIE) Conference Series. Vol. 9144 of Society of Photo-Optical Instrumentation Engineers (SPIE) Conference Series. p. 4.

Kostelecký, V. A., Mewes, M., May 2013. Constraints on Relativity Violations from Gamma-Ray Bursts. Physical Review Letters 110 (20), 201601.

Krawczynski, H., 2011. Analysis of the data from Compton X-ray polarimeters which measure the azimuthal and polar scattering angles. Astropart. Phys. 34, 784-788.

Krawczynski, H., Jan. 2012. The Polarization Properties of Inverse Compton Emission and Implications for Blazar Observations with the GEMS X-Ray Polarimeter. ApJ744, 30.

Krawczynski, H., Angelini, L., Baring, M., et al., Mar. 2013. White Paper for Blazar Observations with a GEMS-like X-ray Polarimetry Mission. ArXiv e-prints.

Krawczynski, H., Garson, A., Guo, Q., et al., Feb. 2011. Scientific prospects for hard X-ray polarimetry. Astroparticle Physics 34, 550-567.

Kuiper, L., Hermsen, W., Mendez, M., Oct. 2004. Discovery of Hard Nonthermal Pulsed X-Ray Emission from the Anomalous X-Ray Pulsar 1E 1841-045. ApJ613, 1173-1178.

Lai, D., Ho, W. C. G., May 2003. Transfer of Polarized Radiation in Strongly Magnetized Plasmas and Thermal Emission from Magnetars: Effect of Vacuum Polarization. ApJ588, 962-974.

Laurent, P., Rodriguez, J., Wilms, J., et al., Apr. 2011. Polarized Gamma-Ray Emission from the Galactic Black Hole Cygnus X-1. Science 332, 438-.

Lei, F., Dean, A. J., Hills, G. L., Nov. 1997. Compton Polarimetry in Gamma-Ray Astronomy. Space Science Review82, 309-388.

Levine, A. M., Bradt, H., Cui, W., et al., Sep. 1996. First Results from the All-Sky Monitor on the Rossi X-Ray Timing Explorer. ApJL469, L33.

Li, L.-X., Narayan, R., McClintock, J. E., Jan. 2009. Inferring the Inclination of a Black Hole Accretion Disk from Observations of its Polarized Continuum Radiation. ApJ691, 847-865.

Liebe, C. C., Craig, W., Kim, Y., et al., Apr. 2012. Calibration and alignment of metrology system for the Nuclear Spectroscopic Telescope Array mission. Optical Engineering 51 (4), 043605.

Lodato, G., Price, D. J., Jun. 2010. On the diffusive propagation of warps in thin accretion discs. MNRAS405, 1212-1226.

Lyubarsky, Y. E., Dec. 2012. Highly magnetized region in pulsar wind nebulae and origin of the Crab gamma-ray 
flares. MNRAS427, 1497-1502.

Madsen, K. K., Harrison, F. A., Markwardt, C., et al., Apr. 2015. Calibration of the NuSTAR High Energy Focusing X-ray Telescope. ArXiv e-prints.

Marinucci, A., Matt, G., Kara, E., et al., May 2014a. Simultaneous NuSTAR and XMM-Newton 0.5-80 keV spectroscopy of the narrow-line Seyfert 1 galaxy SWIFT J2127.4+5654. MNRAS440, 2347-2356.

Marinucci, A., Matt, G., Miniutti, G., et al., May 2014b. The Broadband Spectral Variability of MCG-6-30-15 Observed by NuSTAR and XMM-Newton. ApJ787, 83. Marscher, A. P., Jorstad, S. G., D'Arcangelo, F. D., et al., Apr. 2008. The inner jet of an active galactic nucleus as revealed by a radio-to- $\gamma$-ray outburst. Nature $452,966-$ 969.

McGlynn, S., Clark, D. J., Dean, A. J., et al., May 2007. Polarisation studies of the prompt gamma-ray emission from GRB 041219a using the spectrometer aboard INTEGRAL. A\&A466, 895-904.

McKinney, J. C., Jun. 2006. General relativistic magnetohydrodynamic simulations of the jet formation and large-scale propagation from black hole accretion systems. MNRAS368, 1561-1582.

McKinney, J. C., Blandford, R. D., Mar. 2009. Stability of relativistic jets from rotating, accreting black holes via fully three-dimensional magnetohydrodynamic simulations. MNRAS394, L126-L130.

McKinney, J. C., Tchekhovskoy, A., Blandford, R. D., Jan. 2013. Alignment of Magnetized Accretion Disks and Relativistic Jets with Spinning Black Holes. Science $339,49-$.

McKinney, J. C., Tchekhovskoy, A., Sadowski, A., Narayan, R., Jul. 2014. Three-dimensional general relativistic radiation magnetohydrodynamical simulation of super-Eddington accretion, using a new code HARMRAD with M1 closure. MNRAS441, 3177-3208.

Mészáros, P., 1992. High-energy radiation from magnetized neutron stars. The University of Chicago Press.

Meszaros, P., Novick, R., Szentgyorgyi, A., Chanan, G. A., Weisskopf, M. C., Jan. 1988. Astrophysical implications and observational prospects of X-ray polarimetry. ApJ324, 1056-1067.

Migliari, S., Fender, R. P., Feb. 2006. Jets in neutron star X-ray binaries: a comparison with black holes. MNRAS366, 79-91.

Miniutti, G., Fabian, A. C., Goyder, R., Lasenby, A. N., Sep. 2003. The lack of variability of the iron line in MCG-6-30-15: general relativistic effects. MNRAS344, L22-L26.

Moran, P., Shearer, A., Mignani, R. P., et al., Aug. 2013. Optical polarimetry of the inner Crab nebula and pulsar. MNRAS433, 2564-2575.

Morgan, E. H., Remillard, R. A., Greiner, J., Jun. 1997. RXTE Observations of QPOs in the Black Hole Candidate GRS 1915+105. ApJ482, 993-1010.

Mori, K., Gotthelf, E. V., Zhang, S., et al., Jun. 2013.
NuSTAR Discovery of a $3.76 \mathrm{~s}$ Transient Magnetar Near Sagittarius A*. ApJL770, L23.

Motta, S. E., Casella, P., Henze, M., et al., Feb. 2015. Geometrical constraints on the origin of timing signals from black holes. MNRAS447, 2059-2072.

National Aeronautics and Space Administration, 2014. Announcement of opportunity for astrophysics small explorer mission, solicitation number: Nnh14zda013o. URL https://www.fbo.gov/

Novick, R., Dec. 1975. Stellar and Solar X-Ray Polarimetry. Space Science Review18, 389-408.

Özel, F., Dec. 2001. Surface Emission Properties of Strongly Magnetic Neutron Stars. ApJ563, 276-288.

Papadakis, I. E., Kazanas, D., Akylas, A., Oct. 2005. Fourier-Resolved Spectroscopy of the XMM-Newton Observations of MCG -06-30-15. ApJ631, 727-732.

Parker, M. L., Wilkins, D. R., Fabian, A. C., et al., Sep. 2014. The NuSTAR spectrum of Mrk 335: extreme relativistic effects within two gravitational radii of the event horizon? MNRAS443, 1723-1732.

Ramsey, B. f., 2014. IXPE - The Imaging X-ray Polarimetry Explorer.

Rana, V. R., Cook, III, W. R., Harrison, F. A., Mao, P. H., Miyasaka, H., Aug. 2009. Development of focal plane detectors for the Nuclear Spectroscopic Telescope Array (NuSTAR) mission. In: Society of Photo-Optical Instrumentation Engineers (SPIE) Conference Series. Vol. 7435 of Society of Photo-Optical Instrumentation Engineers (SPIE) Conference Series. p. 3.

Reid, M. J., McClintock, J. E., Steiner, J. F., et al., Nov. 2014. A Parallax Distance to the Microquasar GRS $1915+105$ and a Revised Estimate of its Black Hole Mass. ApJ796, 2.

Remillard, R. A., McClintock, J. E., Sep. 2006. X-Ray Properties of Black-Hole Binaries. ARA\&A44, 49-92.

Reynolds, C. S., Sep. 2014. Measuring Black Hole Spin Using X-Ray Reflection Spectroscopy. Space Science Review183, 277-294.

Risaliti, G., Harrison, F. A., Madsen, K. K., et al., Feb. 2013. A rapidly spinning supermassive black hole at the centre of NGC 1365. Nature494, 449-451.

Rodriguez, J., Grinberg, V., Laurent, P., et al., Jul. 2015. Spectral State Dependence of the 0.4-2 MeV Polarized Emission in Cygnus X-1 Seen with INTEGRAL/IBIS, and Links with the AMI Radio Data. ApJ807, 17.

Ross, R. R., Fabian, A. C., Mar. 1993. The effects of photoionization on X-ray reflection spectra in active galactic nuclei. MNRAS261, 74-82.

Rudy, A., Horns, D., DeLuca, A., et al., Apr. 2015. Characterization of the Inner Knot of the Crab: The Site of the Gamma-ray Flares? ArXiv e-prints.

Ryan, B. R., Dolence, J. C., Gammie, C. F., Jul. 2015. bhlight: General Relativistic Radiation Magnetohydrodynamics with Monte Carlo Transport. ApJ807, 31.

Sadowski, A., Narayan, R., Aug. 2015a. Photonconserving Comptonization in simulations of accretion 
disks around black holes. ArXiv e-prints.

Sadowski, A., Narayan, R., Mar. 2015b. Powerful radiative jets in super-critical accretion disks around nonspinning black holes. ArXiv e-prints.

Sanchez Almeida, J., Martinez Pillet, V., Aug. 1993. Polarizing properties of grazing-incidence X-ray mirrors Comment. Applied Optics 32, 4231-4235.

Sądowski, A., Narayan, R., Tchekhovskoy, A., Zhu, Y., Mar. 2013. Semi-implicit scheme for treating radiation under M1 closure in general relativistic conservative fluid dynamics codes. MNRAS429, 3533-3550.

Schnittman, J. D., Krolik, J. H., Aug. 2009. X-ray Polarization from Accreting Black Holes: The Thermal State. ApJ701, 1175-1187.

Schnittman, J. D., Krolik, J. H., Apr. 2010. X-ray Polarization from Accreting Black Holes: Coronal Emission. ApJ712, 908-924.

Schnittman, J. D., Krolik, J. H., Nov. 2013. A Monte Carlo Code for Relativistic Radiation Transport around Kerr Black Holes. ApJ777, 11.

Schnittman, J. D., Krolik, J. H., Noble, S. C., Jun. 2013. X-Ray Spectra from Magnetohydrodynamic Simulations of Accreting Black Holes. ApJ769, 156.

Shapiro, S. L., Lightman, A. P., Eardley, D. M., Feb. 1976. A two-temperature accretion disk model for Cygnus X-1 - Structure and spectrum. ApJ204, 187-199.

Shaviv, N. J., Heyl, J. S., Lithwick, Y., Jun. 1999. Magnetic lensing near ultramagnetized neutron stars. MNRAS306, 333-347.

Shcherbakov, R. V., Penna, R. F., McKinney, J. C., Aug. 2012. Sagittarius A* Accretion Flow and Black Hole Parameters from General Relativistic Dynamical and Polarized Radiative Modeling. ApJ755, 133.

Silver, E. H., Weisskopf, M. C., Kestenbaum, H. L., et al., Aug. 1979. The first search for X-ray polarization in the Centaurus X-3 and Hercules X-1 pulsars. ApJ232, 248-254.

Soffitta, P., Barcons, X., Bellazzini, R., et al., Dec. 2013. XIPE: the X-ray imaging polarimetry explorer. Experimental Astronomy 36, 523-567.

Spruit, H. C., Sep. 2011. Magnetically powered jets. In: Aharonian, F. A., Hofmann, W., Rieger, F. M. (Eds.), American Institute of Physics Conference Series. Vol. 1381 of American Institute of Physics Conference Series. pp. 227-246.

Stokes, G. G., 1852. Composition and resolution of streams of polarized light from multiple sources. Trans. Cambridge Philos. Soc. 9, 399-416, reprinted in Mathematical and Physical Papers, Vol. 3, Cambridge University Press, London, 1901.

Sunyaev, R. A., Thorne, K. S., 1973. Discussion in Moscow, see Thorne \& Price (1973).

Sunyaev, R. A., Titarchuk, L. G., Jun. 1980. Comptonization of X-rays in plasma clouds - Typical radiation spectra. A\&A86, 121-138.

Sunyaev, R. A., Titarchuk, L. G., Feb. 1985. Comptoniza- tion of low-frequency radiation in accretion disks Angular distribution and polarization of hard radiation. A\&A143, 374-388.

Takeuchi, Y., Kitaguchi, T., Hayato, A., et al., Jul. 2014. Properties of the flight model gas electron multiplier for the GEMS mission. In: Society of Photo-Optical Instrumentation Engineers (SPIE) Conference Series. Vol. 9144 of Society of Photo-Optical Instrumentation Engineers (SPIE) Conference Series. p. 4.

Tavani, M., Bulgarelli, A., Vittorini, V., et al., Feb. 2011. Discovery of Powerful Gamma-Ray Flares from the Crab Nebula. Science 331, 736-.

Taverna, R., Muleri, F., Turolla, R., et al., Feb. 2014. Probing magnetar magnetosphere through X-ray polarization measurements. MNRAS438, 1686-1697.

Tchekhovskoy, A., Narayan, R., McKinney, J. C., Nov. 2011. Efficient generation of jets from magnetically arrested accretion on a rapidly spinning black hole. MNRAS418, L79-L83.

Tendulkar, S. P., Fürst, F., Pottschmidt, K., et al., Nov. 2014. NuSTAR Discovery of a Cyclotron Line in the Be/X-Ray Binary RX J0520.5-6932 during Outburst. ApJ795, 154.

The European Space Agency, 2015. Three Candidates for ESA's Next Medium Class Science Mission.

URL http://www.esa.int/Our_Activities /Space_Science/Three_candidates_for_ESA_s_next medium-class_science_mission

The National Aeronautics and Space Administration, 2015. Three Candidates for ESA's Next Medium Class Science Mission.

URL http://www. nasa.gov/press-release /nasa-selects-proposals-to-study-neutronstars-black-holes-and-more

Thompson, C., Duncan, R. C., Jul. 1995. The soft gamma repeaters as very strongly magnetized neutron stars - I. Radiative mechanism for outbursts. MNRAS 275, 255300.

Thorne, K. S., Price, R. H., Feb. 1975. Cygnus X-1 an interpretation of the spectrum and its variability. ApJL195, L101-L105.

Tomsick, J. A., Kaaret, P., Feb. 2001. The X-Ray Properties of Low-Frequency Quasi-periodic Oscillations from GRS 1915+105 Up to $120 \mathrm{KEV}$. ApJ548, 401-409.

Tomsick, J. A., Nowak, M. A., Parker, M., et al., Jan. 2014. The Reflection Component from Cygnus X-1 in the Soft State Measured by NuSTAR and Suzaku. ApJ780, 78.

Tosti, G., Fiorucci, M., Luciani, M., et al., Nov. 1998. Radio, optical and photopolarimetric observations of Markarian 421 around the great 1996-97 outburst. A\&A339, 41-51.

Trümper, J. E., Dennerl, K., Kylafis, N. D., Ertan, Ü., Zezas, A., Feb. 2013. An Accretion Model for the Anomalous X-Ray Pulsar 4U 0142+61. ApJ764, 49.

Trümper, J. E., Zezas, A., Ertan, Ü., Kylafis, N. D., Jul. 
2010. The energy spectrum of anomalous X-ray pulsars and soft gamma-ray repeaters. A\&A518, A46.

Ubertini, P., Lebrun, F., Di Cocco, G., et al., Nov. 2003. IBIS: The Imager on-board INTEGRAL. A\&A411, L131-L139.

Ueda, Y., Honda, K., Takahashi, H., et al., Apr. 2010. Suzaku Observation of GRS 1915+105: Evolution of Accretion Disk Structure during Limit-cycle Oscillation. ApJ713, 257-268.

Uttley, P., Cackett, E. M., Fabian, A. C., Kara, E., Wilkins, D. R., Aug. 2014. X-ray reverberation around accreting black holes. A\&ARv22, 72 .

Uttley, P., McHardy, I. M., May 2001. The flux-dependent amplitude of broadband noise variability in X-ray binaries and active galaxies. MNRAS323, L26-L30.

Uttley, P., McHardy, I. M., Vaughan, S., May 2005. Nonlinear X-ray variability in X-ray binaries and active galaxies. MNRAS359, 345-362.

Vaughan, S., Fabian, A. C., Nandra, K., Mar. 2003. X-ray continuum variability of MCG-6-30-15. MNRAS339, $1237-1255$.

Vedrenne, G., Roques, J.-P., Schönfelder, V., et al., Nov. 2003. SPI: The spectrometer aboard INTEGRAL. A\&A411, L63-L70.

Vinokur, M., 1965. Optimisation dans la Recherche d'une Sinusode de Priode Connue en Prsence de Bruit. Application a la Radioastronomie. Annales d'Astrophysique $28,412-445$.

Walton, D. J., Risaliti, G., Harrison, F. A., et al., Jun. 2014. NuSTAR and XMM-NEWTON Observations of NGC 1365: Extreme Absorption Variability and a Constant Inner Accretion Disk. ApJ788, 76.

Weisskopf, M. C., Elsner, R. F., Hanna, D., et al., Nov. 2006. The prospects for X-ray polarimetry and its potential use for understanding neutron stars. ArXiv Astrophysics e-prints.

Weisskopf, M. C., Silver, E. H., Kestenbaum, H. L., Long, K. S., Novick, R., Mar. 1978. A precision measurement of the X-ray polarization of the Crab Nebula without pulsar contamination. ApJL220, L117-L121.

Weisskopf, M. C., et al., 2006. The prospects for X-ray polarimetry and its potential use for understanding neutron stars. Paper presented at the 363rd Heraeus Seminar in Bad Honnef, Germany, 2006. arXiv: astroph/0611483v1.

Wijnands, R., van der Klis, M., Apr. 1999. The Broadband Power Spectra of X-Ray Binaries. ApJ514, 939-944.

Willis, D. R., Barlow, E. J., Bird, A. J., et al., Aug. 2005. Evidence of polarisation in the prompt gamma-ray emission from GRB 930131 and GRB 960924. A\&A439, $245-253$.

Wilms, J., Brand, T., Barret, D., et al., Jul. 2014. ATHENA end-to-end simulations. In: Society of PhotoOptical Instrumentation Engineers (SPIE) Conference Series. Vol. 9144 of Society of Photo-Optical Instrumentation Engineers (SPIE) Conference Series. p. 5.
Wolter, H., 1952. Spiegelsysteme streifenden Einfalls als abbildende Optiken für Röntgenstrahlen. Annalen der Physik 445, 94-114.

Yonetoku, D., Murakami, T., Gunji, S., et al., Oct. 2012. Magnetic Structures in Gamma-Ray Burst Jets Probed by Gamma-Ray Polarization. ApJL758, L1.

Yonetoku, D., Murakami, T., Gunji, S., et al., Dec. 2011. Detection of Gamma-Ray Polarization in Prompt Emission of 2011Sci...332..438L2011Sci...332..438L 100826A. ApJL743, L30.

Zhang, H., Böttcher, M., Sep. 2013. X-Ray and GammaRay Polarization in Leptonic and Hadronic Jet Models of Blazars. ApJ774, 18.

Zhang, H., Chen, X., Böttcher, M., Jul. 2014. Synchrotron Polarization in Blazars. ApJ789, 66.

Zhang, H., Chen, X., Böttcher, M., Guo, F., Li, H., May 2015a. Polarization Swings Reveal Magnetic Energy Dissipation in Blazars. ApJ804, 58.

Zhang, L., Chen, L., Qu, J.-l., Bu, Q.-c., Feb. 2015b. The NuSTAR View of a QPO Evolution of GRS 1915+105. AJ149, 82.

Zhang, S.-N., Dec. 2013. Black hole binaries and microquasars. Frontiers of Physics 8, 630-660.

Zhang, W. W., Aug. 2009. Manufacture of mirror glass substrates for the NuSTAR mission. In: Society of Photo-Optical Instrumentation Engineers (SPIE) Conference Series. Vol. 7437 of Society of Photo-Optical Instrumentation Engineers (SPIE) Conference Series. p. 0.

Zhu, Y., Narayan, R., Sadowski, A., Psaltis, D., Aug. 2015. HERO - A 3D general relativistic radiative postprocessor for accretion discs around black holes. MNRAS451, 1661-1681.

Zoghbi, A., Cackett, E. M., Reynolds, C., et al., Jul. 2014. Observations of MCG-5-23-16 with Suzaku, XMMNewton and NuSTAR: Disk Tomography and Compton Hump Reverberation. ApJ789, 56.

Zoghbi, A., Fabian, A. C., Reynolds, C. S., Cackett, E. M., May 2012. Relativistic iron $\mathrm{K}$ X-ray reverberation in NGC 4151. MNRAS422, 129-134. 